\title{
Blow-up and nonexistence of sign changing solutions to the Brezis-Nirenberg problem in dimension three
}

\section{'Blow-up' et non-existence des solutions changeant de signe pour le problème de Brezis-Nirenberg en dimension trois}

\author{
Mohamed Ben Ayed ${ }^{\mathrm{a}}$, Khalil El Mehdi ${ }^{\mathrm{b}}$, Filomena Pacella ${ }^{\mathrm{c}, *}$ \\ a Département de Mathématiques, Faculté des Sciences de Sfax, Route Soukra, Sfax, Tunisia \\ ${ }^{\mathrm{b}}$ Faculté des Sciences et Techniques, Université de Nouakchott, BP 5026, Nouakchott, Mauritania \\ c Dipartimento di Matematica, Universitá di Roma "La Sapienza", P. le A. Moro 2, 00185, Roma, Italy
}

Received 28 February 2005; received in revised form 6 June 2005; accepted 7 July 2005

Available online 6 December 2005

\begin{abstract}
In this paper we study low energy sign changing solutions of the critical exponent problem $\left(\mathrm{P}_{\lambda}\right):-\Delta u=u^{5}+\lambda u$ in $\Omega, u=0$ on $\partial \Omega$, where $\Omega$ is a smooth bounded domain in $\mathbb{R}^{3}$ and $\lambda$ is a real positive parameter. We make a precise blow-up analysis of this kind of solutions and prove some comparison results among some limit values of the parameter $\lambda$ which are related to the existence of positive or of sign changing solutions.

(c) 2006 L'Association Publications de l'Institut Henri Poincaré. Published by Elsevier B.V. All rights reserved
\end{abstract}

\section{Résumé}

Dans cet article, nous étudions les solutions changeant de signe et à énergie minimale du problème avec exposant critique $\left(\mathrm{P}_{\lambda}\right):-\Delta u=u^{5}+\lambda u$ dans $\Omega, u=0$ sur $\partial \Omega$, où $\Omega$ est un domaine borné et régulier de $\mathbb{R}^{3}$ et $\lambda$ est un paramètre réel strictement positif. Nous faisons une analyse précise du 'blow-up' de ce type de solutions et nous prouvons des résultats de comparaisons pour certaines valeurs limites du paramètre $\lambda$ qui sont liées à l'existence des solutions positives ou des solutions changeant de signe. (C) 2006 L'Association Publications de l'Institut Henri Poincaré. Published by Elsevier B.V. All rights reserved

MSC: $35 \mathrm{~J} 20 ; 35 \mathrm{~J} 60$

Keywords: Blow-up analysis; Sign changing solutions; Nodal domains; Critical exponent

Mots-clés : Analyse du 'blow-up' ; Solutions changeant de signe ; Domaines nodaux ; Exposant critique

\footnotetext{
ฟ M. Ben Ayed and F. Pacella are supported by MURST, Project "Metodi Variazionali ed Equazioni Differenziali non lineari”. K. El Mehdi is supported by Istituto Nazionale di Alta Matematica.

* Corresponding author.

E-mail addresses: Mohamed.Benayed@fss.rnu.tn (M. Ben Ayed), khalil@univ-nkc.mr (K. El Mehdi), pacella@mat.uniroma1.it (F. Pacella).
} 


\section{Introduction}

In this paper we study low energy sign changing solutions of the problem known as the Brezis-Nirenberg problem in smooth bounded domains in $\mathbb{R}^{3}$. We get two kind of results. First with simple arguments we prove some comparison results among some limit values of the parameter $\lambda$ which are connected with the existence of certain kind of positive or sign changing solutions. Second we make a precise blow-up analysis of sign changing solutions whose energy converges to the value $2 S^{3 / 2}, S$ being the best Sobolev constant for the embedding of $H_{0}^{1}(\Omega)$ into $L^{6}(\Omega)$.

To be more precise we need some notations and recall previous results.

Let us consider the Brezis-Nirenberg problem which is the following elliptic problem with critical nonlinearity

$$
\begin{cases}-\Delta u=|u|^{2^{*}-2} u+\lambda u & \text { in } \Omega, \\ u=0 & \text { on } \partial \Omega,\end{cases}
$$

where $\Omega$ is a smooth bounded domain in $\mathbb{R}^{n}, n \geqslant 3, \lambda$ is a real positive parameter and $2^{*}=2 n /(n-2)$ is the critical Sobolev exponent for the embedding of $H_{0}^{1}(\Omega)$ into $L^{2^{*}}(\Omega)$.

About twenty years ago Brezis and Nirenberg in the celebrated paper [8] proved that if $n \geqslant 4$ there exists a positive solution of (1.1) for every $\lambda \in\left(0, \lambda_{1}(\Omega)\right), \lambda_{1}(\Omega)$ being the first eigenvalue of $-\Delta$ on $\Omega$. Such a positive solution is a minimizer of the functional

$$
J_{\lambda}(u)=\frac{\int_{\Omega}|\nabla u|^{2}-\lambda \int_{\Omega}|u|^{2}}{\left(\int_{\Omega}|u|^{2^{*}}\right)^{2 / 2^{*}}}
$$

in the space $H_{0}^{1}(\Omega)$. The three-dimensional case is quite different. In this case let us rewrite problem (1.1) as

$$
\left(\mathrm{P}_{\lambda}\right) \quad \begin{cases}-\Delta u=u^{5}+\lambda u & \text { in } \Omega, \\ u=0 & \text { on } \partial \Omega .\end{cases}
$$

Unlike the case of higher dimensions, when $\Omega$ is a ball $B$ in $\mathbb{R}^{3}$ Brezis and Nirenberg [8] proved that a positive solution of $\left(\mathrm{P}_{\lambda}\right)$ exists if and only if $\lambda \in\left(\frac{\lambda_{1}(B)}{4}, \lambda_{1}(B)\right)$. In view of [1] the positive solution is unique and is indeed the minimum of (1.2). For more general bounded domains they proved that if $\Omega$ is strictly starshaped about the origin then, defining

$$
\lambda_{0}(\Omega)=\inf \{\lambda \in \mathbb{R} \mid(1.1) \text { has a positive solution }\},
$$

it results $\lambda_{0}(\Omega)>0$.

Another important number connected with the existence of positive solutions is the following

$$
\lambda^{*}(\Omega)=\inf \{\lambda \in \mathbb{R} \mid \text { a minimizer for (1.2) exists }\} .
$$

Note that, by Remark 2.3 in Section 2, we have

$$
\lambda^{*}(\Omega)=\inf \left\{\lambda \in \mathbb{R} \mid\left(\mathrm{P}_{\lambda}\right) \text { has a positive solution } u_{\lambda} \text { with }\left\|u_{\lambda}\right\|_{\lambda}^{2} \leqslant S^{3 / 2}\right\},
$$

where $S$ denotes the Sobolev constant, that is, $S=\inf _{u \in H_{0}^{1}(\Omega), u \neq 0}\left(\|u\|^{2} /\|u\|_{L^{6}(\Omega)}^{2}\right)$, with $\|u\|^{2}=\int_{\Omega}|\nabla u|^{2}$ and where

$$
\left\|u_{\lambda}\right\|_{\lambda}^{2}=\int_{\Omega}|\nabla u|^{2}-\lambda \int_{\Omega} u^{2} .
$$

As recalled before, by [8], we have that $\lambda^{*}(B)=\lambda_{0}(B)=\lambda_{1}(B) / 4$ in the case of the ball.

Obviously for general domains $\lambda_{0}(\Omega) \leqslant \lambda^{*}(\Omega)$ and, as far as we know, is not yet clear in which cases $\lambda_{0}(\Omega)=$ $\lambda^{*}(\Omega)$.

A recent interesting result which states the equivalence between the existence of a minimizer for (1.2) and the fact that the infimum is strictly smaller than $S$ and as well relates the existence of a minimizer to the fact that the regular part of the Green function of the operator $(-\Delta-\lambda)$ becomes negative at some point of the domain $\Omega$ is contained in [11].

Concerning the existence of sign changing solutions of (1.1), several results have been obtained if $n \geqslant 4$. As expected, in this case one can get sign changing solutions for every $\lambda \in\left(0, \lambda_{1}(\Omega)\right)$ or even for $\lambda>\lambda_{1}(\Omega)$. For details one can see the papers by Atkinson, Brezis and Peletier [2,3], Clapp and Weth [10] and the references therein. 
Obviously the case $n=3$ presents the same difficulties enlightened in [8] for positive solutions.

In the first part of this paper, with a simple argument, we prove that there cannot exist sign changing solutions with low energy in correspondence to a value of the parameter $\lambda$ smaller than $\lambda^{*}(\Omega)$.

More precisely, let us define

$$
\bar{\lambda}(\Omega)=\inf \left\{\lambda \in \mathbb{R} \mid\left(\mathrm{P}_{\lambda}\right) \text { has a sign changing solution } u_{\lambda} \text { with }\left\|u_{\lambda}\right\|_{\lambda}^{2} \leqslant 2 S^{3 / 2}\right\} .
$$

Note that sign changing solutions with energy smaller than $2 S^{3 / 2}$ always exist if $\Omega$ is a ball as it can be easily seen by considering the positive solution in the half ball which minimizes (1.2) and extending it by oddness to the whole ball.

It should also be pointed out that solutions to (1.1) are smooth as a consequence of a result due to Brezis and Kato [7] (see also [6]).

Now, we state our comparison results:

Theorem 1.1. Let $\lambda$ be such that there exists a nontrivial solution $u_{\lambda}$ of $\left(\mathrm{P}_{\lambda}\right)$ satisfying:

there exists a connected component $\Omega_{1}$ of $\Omega \backslash\left\{x \in \Omega \mid u_{\lambda}(x)=0\right\}$ such that

$$
\frac{\int_{\Omega_{1}}\left|\nabla u_{\lambda}\right|^{2}-\lambda \int_{\Omega_{1}}\left|u_{\lambda}\right|^{2}}{\left(\int_{\Omega_{1}}\left|u_{\lambda}\right|^{2^{*}}\right)^{2 / 2^{*}}} \leqslant S .
$$

Then we have

$$
\lambda \geqslant \lambda^{*}(\Omega)
$$

In the case of sign changing solutions this theorem essentially claims that if $\lambda<\lambda^{*}(\Omega)$ there cannot exist a sign changing solution $u_{\lambda}$ with a nodal region $\Omega_{1}$ in which the energy of $u_{\lambda \mid \Omega_{1}}$ is smaller than or equal to $S^{3 / 2}$.

Corollary 1.2. We have that

$$
\bar{\lambda}(\Omega) \geqslant \lambda^{*}(\Omega) \text {. }
$$

Corollary 1.3. If $\bar{\lambda}(\Omega)$ is achieved, then we have an alternative:

either

$$
\bar{\lambda}(\Omega)>\lambda^{*}(\Omega)
$$

or

$$
\bar{\lambda}(\Omega)=\lambda^{*}(\Omega) \text { and hence } \lambda^{*}(\Omega) \text { is achieved. }
$$

In particular, if $\Omega$ is a ball $B$ then

$$
\bar{\lambda}(B)>\lambda_{1}(B) / 4 \text {. }
$$

Another result that is obtained with same proof as for Theorem 1.1 is the following

Corollary 1.4. Assume that $\Omega$ is symmetric with respect to the plane $T=\left\{x=\left(x_{1}, x_{2}, x_{3}\right) \in \mathbb{R}^{3} \mid x_{1}=0\right\}$ and let $D_{1}$ be the set $D_{1}=\left\{x \in \Omega \mid x_{1}<0\right\}$. Then we have

$$
\bar{\lambda}(\Omega) \leqslant \lambda^{*}\left(D_{1}\right) \text { and } \quad \lambda^{*}\left(D_{1}\right) \geqslant \lambda^{*}(\Omega) \text {. }
$$

Though it applies only to symmetric domains, the result of Corollary 1.4 indicates a kind of monotonicity of the parameter $\lambda^{*}(\Omega)$ with respect to the domain $\Omega$ as it happens for the first Dirichlet eigenvalue of $-\Delta$ on $\Omega$. In view of the result of [11] it also gives a relation between the sign of the regular part of the Green function of the operator $(-\Delta-\lambda)$ in $\Omega$ and in $D_{1}$.

Theorem 1.1 and Corollaries 1.2 and 1.3 are also related to the following question raised by H. Brezis If $\Omega$ is a ball $B$ in $\mathbb{R}^{3}$, could exist sign changing solutions of $\left(\mathrm{P}_{\lambda}\right)$ when $\lambda$ is smaller than $\lambda^{*}(B)=\lambda_{1}(B) / 4$ ? 
Obviously the above results give a very partial answer to the above question since they only concern the case of sign changing solutions $u_{\lambda}$ with a nodal region $\Omega_{1}$ in which the energy $\left\|u_{\lambda}\right\|_{\lambda}^{2}$ is not bigger than $S^{3 / 2}$.

Note that if $\bar{\lambda}(\Omega)$ was achieved by a sign changing solution $u_{\lambda}$ then either the energy of $u_{\lambda}$ should be less than $2 S^{3 / 2}$ and $u_{\lambda}$ should be degenerate otherwise it could be continued to a solution $u_{\lambda^{\prime}}, \lambda^{\prime}<\bar{\lambda}(\Omega)$, contradicting the definition of $\bar{\lambda}(\Omega)$, or the energy of $u_{\lambda}$ should be equal to $2 S^{3 / 2}$.

In the case when $\bar{\lambda}(\Omega)$ is not achieved it is possible to prove (see Lemma 4.2 below) that there exists a family of solutions $u_{\lambda}$ such that $\left\|u_{\lambda}\right\|^{2} \rightarrow 2 S^{3 / 2}$ and $u_{\lambda} \rightarrow 0$ in $H_{0}^{1}(\Omega)$, as $\lambda \rightarrow \bar{\lambda}(\Omega)$, i.e. the solutions $u_{\lambda}$ concentrate. Hence it is an interesting question to analyze the concentration phenomenon of these solutions.

Indeed the second part of the paper is devoted to analyze the behavior of sign changing solutions of $\left(\mathrm{P}_{\lambda}\right)$ which converge weakly to zero and whose energy converges to $2 S^{3 / 2}$ as $\lambda \rightarrow \bar{\lambda}(\Omega)$. More precisely we prove that these solutions blow-up at two points $\bar{a}_{1}$ and $\bar{a}_{2}$ which are the limit of the concentration points $a_{\lambda, 1}$ and $a_{\lambda, 2}$ of the positive and negative part of the solutions. Moreover the distance between $a_{\lambda, 1}$ and $a_{\lambda, 2}$ is bounded from below by a positive constant depending only on $\Omega$ and the speeds of concentrations of the positive and negative part are comparable. We think that these results, whose precise statements are contained in Theorems 3.1 and 4.1 below, are interesting in theirselves and important to face the study of sign changing solutions of problems with critical Sobolev exponent. They were also difficult to get since we could not always exploit the same arguments used in the study of positive solutions blowing up at two points.

It is also interesting that, once the blow-up analysis is carried out, we can give an alternative proof of Corollary 1.2, in the case when $\bar{\lambda}(\Omega)$ is not achieved, which relies on Pohozaev's identity and on the sign of the regular part of the Green function of $(-\Delta-\bar{\lambda}(\Omega))$ as deduced by the result of [11].

A final comment is that one expects that results analogous to those of Theorems 3.1 and 4.1 below should also hold in higher dimensions and with similar or even simpler proof. Surprisingly our proof only works in dimension 3 because in applying Pohozaev's identity and getting the convergence of certain integrals the role of the dimension is crucial. We think that other arguments could be used for $n \geqslant 4$ and a further investigation in this direction is in progress.

The outline of the paper is the following. In Section 2 we prove Theorem 1.1 and its corollaries and we also show a qualitative result which gives the connection between the energy of a solution and the number of its nodal regions. Section 3 is devoted to state and prove Theorem 3.1. In Section 4 we state and prove Theorem 4.1 and we then give another proof of Corollary 1.2 in the case when $\bar{\lambda}(\Omega)$ is not achieved. Finally, Appendix A is devoted to the local blow-up analysis needed in Sections 3 and 4.

\section{Proof of Theorem 1.1 and its corollaries}

We start by proving Theorem 1.1.

Proof of Theorem 1.1. First, if $u_{\lambda}$ does not change sign we deduce that $\Omega_{1}=\Omega$. In this case, it is easy to see that

$$
J_{\lambda}\left(u_{\lambda}\right) \leqslant S \text {. }
$$

Assume that

$$
\inf _{u \in H_{0}^{1}(\Omega), u \neq 0} J_{\lambda}(u)=S,
$$

then $u_{\lambda}$ is a minimizer for $J_{\lambda}$ which contradicts the result of [11] according to which the infimum is achieved if and only if it is smaller than $S$. Thus

$$
\inf _{u \in H_{0}^{1}(\Omega), u \neq 0} J_{\lambda}(u)<S
$$

and hence again by the result of [11], $J_{\lambda}$ has a minimizer $v_{\lambda}$. Thus $\lambda \geqslant \lambda^{*}(\Omega)$.

Now, we analyze the case when $u_{\lambda}$ changes sign. In this case, we define $w_{\lambda}$ as

$$
w_{\lambda}=u_{\lambda} \quad \text { in } \Omega_{1}, \quad w_{\lambda}=0 \quad \text { in } \Omega \backslash \Omega_{1} .
$$

Without loss of generality, we can assume that $w_{\lambda} \geqslant 0$. Since $u_{\lambda}=0$ on $\partial \Omega_{1}$, we have $w_{\lambda} \in H_{0}^{1}(\Omega)$. Now, multiplying $\left(\mathrm{P}_{\lambda}\right)$ by $w_{\lambda}$ and integrating on $\Omega$, we obtain 


$$
\left\|w_{\lambda}\right\|_{\lambda}^{2}:=\int_{\Omega}\left|\nabla w_{\lambda}\right|^{2}-\lambda \int_{\Omega} w_{\lambda}^{2}=\int_{\Omega} w_{\lambda}^{6} .
$$

Observe that, because of the assumption (1.6)

$$
J_{\lambda}\left(w_{\lambda}\right):=\frac{\left\|w_{\lambda}\right\|_{\lambda}^{2}}{\left(\int_{\Omega} w_{\lambda}^{6}\right)^{1 / 3}} \leqslant S .
$$

Assume that

$$
\inf _{u \in H_{0}^{1}(\Omega), u \neq 0} J_{\lambda}(u)=S,
$$

then $w_{\lambda}$ is a minimizer for $J_{\lambda}$, which is a contradiction since, by [11], the infimum can only be achieved if it is strictly smaller than $S$. Thus

$$
\inf _{u \in H_{0}^{1}(\Omega), u \neq 0} J_{\lambda}(u)<S
$$

and hence by the result of [11], $J_{\lambda}$ has a minimizer $v_{\lambda}$. Thus $\lambda \geqslant \lambda^{*}(\Omega)$. Theorem 1.1 is thereby proved.

Next, we are going to give the proofs of Corollaries 1.2-1.4.

Proof of Corollary 1.2. Let $\lambda \geqslant \bar{\lambda}(\Omega)$ be such that there exists a sign changing solution $u_{\lambda}$ of $\left(\mathrm{P}_{\lambda}\right)$ with $\left\|u_{\lambda}\right\|_{\lambda}^{2} \leqslant$ $2 S^{3 / 2}$. Since $\left\|u_{\lambda}\right\|_{\lambda}^{2}=\left\|u_{\lambda}^{+}\right\|_{\lambda}^{2}+\left\|u_{\lambda}^{-}\right\|_{\lambda}^{2}$, we have that $\left\|u_{\lambda}^{+}\right\|_{\lambda}^{2} \leqslant S^{3 / 2}$ or $\left\|u_{\lambda}^{-}\right\|_{\lambda}^{2} \leqslant S^{3 / 2}$. Then the assumptions of Theorem 1.1 are satisfied and therefore $\lambda \geqslant \lambda^{*}(\Omega)$. Hence the corollary follows.

Proof of Corollary 1.3. By Theorem 1.1, we know that $\bar{\lambda}(\Omega) \geqslant \lambda^{*}(\Omega)$. If $\bar{\lambda}(\Omega)$ is achieved then as in the proof of Corollary 1.2, we obtain $\left\|u_{\bar{\lambda}(\Omega)}^{+}\right\|_{\bar{\lambda}(\Omega)}^{2} \leqslant S^{3 / 2}$ or $\left\|u_{\bar{\lambda}(\Omega)}^{-}\right\|_{\bar{\lambda}(\Omega)}^{2} \leqslant S^{3 / 2}$. So, following the proof of Theorem 1.1, we derive that $J_{\bar{\lambda}(\Omega)}$ has a minimizer. Now, if we assume that $\bar{\lambda}(\Omega)=\lambda^{*}(\Omega)$ we derive that $J_{\lambda^{*}(\Omega)}$ has a minimizer and therefore $\lambda^{*}(\Omega)$ is achieved.

Since when $\Omega$ is a ball $B$ we know by [8] that $\lambda^{*}(B)=\lambda_{1}(B) / 4$ is not achieved we get $\bar{\lambda}(\Omega)>\lambda_{1}(B) / 4$.

Proof of Corollary 1.4. Let $\lambda>\lambda^{*}\left(D_{1}\right)$ such that there exists a positive solution $u_{\lambda}$ of the problem $\left(\mathrm{P}_{\lambda}\right)$ on $D_{1}$ with $\int_{D_{1}}\left|\nabla u_{\lambda}\right|^{2}-\lambda \int_{D_{1}} u_{\lambda}^{2} \leqslant S^{3 / 2}$. Extending $u_{\lambda}$ by oddness with respect to $T$, we can construct a sign changing solution $v_{\lambda}$ of $\left(\mathrm{P}_{\lambda}\right)$ which satisfies

$$
\int_{\Omega}\left|\nabla v_{\lambda}\right|^{2}-\lambda \int_{\Omega} v_{\lambda}^{2}=2\left(\int_{D_{1}}\left|\nabla u_{\lambda}\right|^{2}-\lambda \int_{D_{1}} u_{\lambda}^{2}\right) \leqslant 2 S^{3 / 2} .
$$

Thus $\lambda \geqslant \bar{\lambda}(\Omega)$ and therefore

$$
\lambda^{*}\left(D_{1}\right) \geqslant \bar{\lambda}(\Omega) \text {. }
$$

Corollary 1.4 follows immediately from (2.3) and Corollary 1.2.

We now state and prove a general result on the relation between the energy of a sign changing solution of $\left(\mathrm{P}_{\lambda}\right)$ and the number of its nodal regions.

Proposition 2.1. Assume that $\mu<\lambda_{1}(\Omega)$ and let $u_{\lambda}$ be a sign changing solution of $\left(\mathrm{P}_{\lambda}\right)$ such that

$$
u_{\lambda} \rightarrow 0 \quad \text { as } \lambda \rightarrow \mu \text {. }
$$

Then, we have

$$
\int_{\Omega}\left|\nabla u_{\lambda}\right|^{2} \geqslant k S^{3 / 2}(1+\mathrm{o}(1)),
$$

where $k$ is the number of the connected components of $\Omega \backslash Z_{\lambda}$, with $Z_{\lambda}=\left\{x \in \Omega \mid u_{\lambda}(x)=0\right\}$. 
Proof. Let $\Omega_{1}$ be a connected component of $\Omega \backslash Z_{\lambda}$. We observe that

$$
\begin{cases}-\Delta u_{\lambda}=u_{\lambda}^{5}+\lambda u_{\lambda} & \text { in } \Omega_{1} \\ u_{\lambda}=0 & \text { on } \partial \Omega_{1} .\end{cases}
$$

Multiplying (2.4) by $u_{\lambda}$ and integrating on $\Omega_{1}$, we obtain

$$
\int_{\Omega_{1}}\left|\nabla u_{\lambda}\right|^{2}=\int_{\Omega_{1}} u_{\lambda}^{6}+\lambda \int_{\Omega_{1}} u_{\lambda}^{2} \leqslant \frac{1}{S^{3}}\left(\int_{\Omega_{1}}\left|\nabla u_{\lambda}\right|^{2}\right)^{3}+\frac{\lambda}{\lambda_{1}\left(\Omega_{1}\right)} \int_{\Omega_{1}}\left|\nabla u_{\lambda}\right|^{2},
$$

where $\lambda_{1}\left(\Omega_{1}\right)$ is the first Dirichlet eigenvalue of $-\Delta$ on $\Omega_{1}$.

Notice that $\lambda_{1}\left(\Omega_{1}\right) \geqslant \lambda_{1}(\Omega)$ and therefore (2.5) implies that

$$
\left(1-\frac{\lambda}{\lambda_{1}(\Omega)}\right) \int_{\Omega_{1}}\left|\nabla u_{\lambda}\right|^{2} \leqslant \frac{1}{S^{3}}\left(\int_{\Omega_{1}}\left|\nabla u_{\lambda}\right|^{2}\right)^{3} .
$$

Hence

$$
\int_{\Omega_{1}}\left|\nabla u_{\lambda}\right|^{2} \geqslant c>0 \quad \text { when } \lambda \text { is close to } \mu,
$$

where $c$ is a positive constant which depends only on $\mu$ and $\lambda_{1}(\Omega)$.

On the other hand, since $u_{\lambda} \rightarrow 0$ in $\Omega$ as $\lambda \rightarrow \mu$, we have that $\int_{\Omega_{1}} u_{\lambda}^{2} \rightarrow 0$ and hence

$$
(1+\mathrm{o}(1)) \int_{\Omega_{1}}\left|\nabla u_{\lambda}\right|^{2} \leqslant \frac{1}{S^{3}}\left(\int_{\Omega_{1}}\left|\nabla u_{\lambda}\right|^{2}\right)^{3} .
$$

Thus, by (2.6)

$$
\int_{\Omega_{1}}\left|\nabla u_{\lambda}\right|^{2} \geqslant S^{3 / 2}(1+\mathrm{o}(1))
$$

Therefore

$$
\int_{\Omega}\left|\nabla u_{\lambda}\right|^{2} \geqslant k S^{3 / 2}(1+\mathrm{o}(1)),
$$

where $k$ is the number of the connected components of $\Omega \backslash Z_{\lambda}$. Therefore our proposition is established.

Clearly, Proposition 2.1 implies the following:

Corollary 2.2. Assume that $\bar{\lambda}(\Omega)<\lambda_{1}(\Omega)$ and let $u_{\lambda}$ be a sign changing solution of $\left(\mathrm{P}_{\lambda}\right)$ such that

$$
\int_{\Omega}\left|\nabla u_{\lambda}\right|^{2} \rightarrow 2 S^{3 / 2} \text { and } u_{\lambda} \rightarrow 0 \quad \text { as } \lambda \rightarrow \bar{\lambda}(\Omega) .
$$

Then the set $\Omega \backslash\left\{x \in \Omega \mid u_{\lambda}(x)=0\right\}$ has exactly two connected components.

Before ending this section, let us mention the following remark:

Remark 2.3. Let

$$
\lambda^{\prime}(\Omega)=\inf \left\{\lambda \in \mathbb{R} \mid\left(\mathrm{P}_{\lambda}\right) \text { has a positive solution } u_{\lambda} \text { with }\left\|u_{\lambda}\right\|_{\lambda}^{2} \leqslant S^{3 / 2}\right\},
$$

with $\left\|u_{\lambda}\right\|_{\lambda}^{2}=\int_{\Omega}\left|\nabla u_{\lambda}\right|^{2}-\lambda \int_{\Omega} u_{\lambda}^{2}$. Then we have

$$
\lambda^{\prime}(\Omega)=\lambda^{*}(\Omega) \text {, }
$$

where $\lambda^{*}(\Omega)$ is defined by (1.4).

Proof. It follows from the same proof as for the case of Theorem 1.1 when $u_{\lambda}$ is a positive solution. 


\section{Blow-up analysis}

This section is devoted to the proof of the following result:

Theorem 3.1. Assume that $\bar{\lambda}(\Omega)<\lambda_{1}(\Omega)$ and let $\left(u_{\lambda}\right)$ be a family of sign changing solutions of $\left(\mathrm{P}_{\lambda}\right)$ which satisfies

$$
\left\|u_{\lambda}\right\|^{2}:=\int_{\Omega}\left|\nabla u_{\lambda}\right|^{2} \rightarrow 2 S^{3 / 2} \text { and } u_{\lambda} \rightarrow 0 \quad \text { as } \lambda \rightarrow \bar{\lambda}(\Omega) .
$$

Then, there exist two local extremum points $a_{\lambda, 1}, a_{\lambda, 2}$ of $u_{\lambda}$ and a positive constant $\alpha$, which depends only on $\Omega$, such that

$$
\begin{aligned}
& \left\|u_{\lambda}-P \delta_{\left(a_{\lambda, 1}, \mu_{\lambda, 1}\right)}+P \delta_{\left(a_{\lambda, 2}, \mu_{\lambda, 2}\right)}\right\| \rightarrow 0, \quad \text { as } \lambda \rightarrow \bar{\lambda}(\Omega), \\
& \mu_{\lambda, i}:=3^{-1 / 2} u_{\lambda}\left(a_{\lambda, i}\right)^{2} \rightarrow+\infty, \quad \text { as } \lambda \rightarrow \bar{\lambda}(\Omega), \text { for } i \in\{1,2\}, \\
& d\left(a_{\lambda, i}, \partial \Omega\right) \geqslant \alpha, \quad \text { for } i \in\{1,2\} \quad \text { and } \quad\left|a_{\lambda, 1}-a_{\lambda, 2}\right| \geqslant \alpha \quad \text { for } \lambda \text { close to } \bar{\lambda}(\Omega),
\end{aligned}
$$

where $P \delta_{(a, \mu)}$ denotes the projection of $\delta_{(a, \mu)}$ on $H_{0}^{1}(\Omega)$, that is,

$$
\Delta P \delta_{(a, \mu)}=\Delta \delta_{(a, \mu)} \quad \text { in } \Omega, \quad P \delta_{(a, \mu)}=0 \quad \text { on } \partial \Omega, \quad \text { and } \quad \delta_{(a, \mu)}(x)=\frac{3^{1 / 4} \mu^{1 / 2}}{\left(1+\mu^{2}|x-a|^{2}\right)^{1 / 2}} .
$$

To prove Theorem 3.1, we need some preliminary results. In the sequel we denote by $\left(u_{\lambda}\right)$ the family of solutions which satisfies the assumption of Theorem 3.1.

Lemma 3.2. We have that

$$
\begin{aligned}
& \text { (i) } \int_{\Omega}\left|\nabla u_{\lambda}^{+}\right|^{2} \rightarrow S^{3 / 2}, \quad \int_{\Omega}\left|\nabla u_{\lambda}^{-}\right|^{2} \rightarrow S^{3} / 2 \quad \text { as } \lambda \rightarrow \bar{\lambda}(\Omega), \\
& \text { (ii) } \int_{\Omega}\left(u_{\lambda}^{+}\right)^{6} \rightarrow S^{3 / 2}, \quad \int_{\Omega}\left(u_{\lambda}^{-}\right)^{6} \rightarrow S^{3 / 2} \quad \text { as } \lambda \rightarrow \bar{\lambda}(\Omega),
\end{aligned}
$$

where $u_{\lambda}^{+}=\max \left(u_{\lambda}, 0\right)$ and $u_{\lambda}^{-}=\max \left(0,-u_{\lambda}\right)$.

Proof. Multiplying $\left(\mathrm{P}_{\lambda}\right)$ by $u_{\lambda}^{ \pm}$and integrating on $\Omega$, we obtain

$$
\int_{\Omega}\left|\nabla u_{\lambda}^{+}\right|^{2}=\int_{\Omega}\left(u_{\lambda}^{+}\right)^{6}+\lambda \int_{\Omega}\left(u_{\lambda}^{+}\right)^{2} \text { and } \int_{\Omega}\left|\nabla u_{\lambda}^{-}\right|^{2}=\int_{\Omega}\left(u_{\lambda}^{-}\right)^{6}+\lambda \int_{\Omega}\left(u_{\lambda}^{-}\right)^{2} .
$$

On the other hand, since $u_{\lambda} \rightarrow 0$ in $\Omega$ as $\lambda \rightarrow \bar{\lambda}(\Omega)$, we have

$$
\int_{\Omega}\left(u_{\lambda}^{+}\right)^{2} \rightarrow 0 \text { and } \int_{\Omega}\left(u_{\lambda}^{-}\right)^{2} \rightarrow 0 \quad \text { as } \lambda \rightarrow \bar{\lambda}(\Omega) .
$$

Therefore, arguing as in the proof of Proposition 2.1, we find

$$
\left\|u_{\lambda}^{+}\right\|^{2} \geqslant S^{3 / 2}(1+\mathrm{o}(1)) \text { and }\left\|u_{\lambda}^{-}\right\|^{2} \geqslant S^{3 / 2}(1+\mathrm{o}(1)) .
$$

Clearly

$$
\left\|u_{\lambda}\right\|^{2}=\left\|u_{\lambda}^{+}\right\|^{2}+\left\|u_{\lambda}^{-}\right\|^{2}=2 S^{3 / 2}(1+\mathrm{o}(1)) .
$$

Therefore claim (i) of Lemma 3.2 follows. Claim (ii) follows from (3.1), (3.2) and claim (i).

Lemma 3.3. We have that

$$
M_{\lambda,+}:=\max _{\Omega} u_{\lambda}^{+} \rightarrow+\infty, \quad M_{\lambda,-}:=\max _{\Omega} u_{\lambda}^{-} \rightarrow+\infty \quad \text { as } \lambda \rightarrow \bar{\lambda}(\Omega) .
$$


Proof. Arguing by contradiction, we assume that $M_{\lambda,+} \leqslant c$ as $\lambda \rightarrow \bar{\lambda}(\Omega)$. Therefore $u_{\lambda}^{+} \in L^{\infty}(\Omega)$ and $u_{\lambda}^{+} \rightarrow 0$ a.e. Thus $\left(u_{\lambda}^{+}\right)^{6} \rightarrow 0$ in $L^{1}(\Omega)$ which contradicts Lemma 3.2. Hence $M_{\lambda,+} \rightarrow \infty$ as $\lambda \rightarrow \bar{\lambda}(\Omega)$.

In the same way, we prove that $M_{\lambda,-} \rightarrow+\infty$ as $\lambda \rightarrow \bar{\lambda}(\Omega)$.

Without loss of generality, we can assume in the sequel that

$$
M_{\lambda,+} \geqslant M_{\lambda,-} \text {. }
$$

Let $\Omega_{\lambda}:=M_{\lambda,+}^{2}\left(\Omega-a_{\lambda, 1}\right)$, where $a_{\lambda, 1} \in \Omega$ such that $M_{\lambda,+}=u_{\lambda}\left(a_{\lambda, 1}\right)$, and we denote by $v_{\lambda}$ the function defined on $\Omega_{\lambda}$ by

$$
v_{\lambda}(y)=M_{\lambda,+}^{-1} u_{\lambda}\left(a_{\lambda, 1}+M_{\lambda,+}^{-2} y\right) .
$$

It is easy to see that $v_{\lambda}$ satisfies

$$
\left(\mathrm{Q}_{\lambda}\right) \quad \begin{cases}-\Delta v_{\lambda}=v_{\lambda}^{5}+\lambda M_{\lambda,+}^{-4} v_{\lambda} & \text { in } \Omega_{\lambda} \\ v_{\lambda}=0 & \text { on } \partial \Omega_{\lambda}, \\ v_{\lambda}(0)=1, \quad\left|v_{\lambda}\right| \leqslant 1 & \text { in } \Omega_{\lambda} .\end{cases}
$$

(Recall that, we have assumed $M_{\lambda,+} \geqslant M_{\lambda,-}$.)

Using the assumptions of Theorem 3.1, we see that

$$
\int_{\Omega_{\lambda}}\left|\nabla v_{\lambda}\right|^{2}=\int_{\Omega}\left|\nabla u_{\lambda}\right|^{2} \rightarrow 2 S^{3 / 2}, \quad \int_{\Omega_{\lambda}}\left|v_{\lambda}\right|^{6}=\int_{\Omega}\left|u_{\lambda}\right|^{6} \rightarrow 2 S^{3 / 2} \quad \text { as } \lambda \rightarrow \bar{\lambda}(\Omega) .
$$

Let us prove the following lemma.

Lemma 3.4. We have that

$$
M_{\lambda,+}^{2} d\left(a_{\lambda, 1}, \partial \Omega\right) \rightarrow+\infty \quad \text { as } \lambda \rightarrow \bar{\lambda}(\Omega) .
$$

Proof. As in the proof of Lemma 2.3 of [5], we can show that

$$
l:=\lim _{\lambda \rightarrow \bar{\lambda}(\Omega)} M_{\lambda,+}^{2} d\left(a_{\lambda, 1}, \partial \Omega\right)>0 .
$$

Arguing by contradiction, we suppose that $l<\infty$. Then it follows from $\left(\mathrm{Q}_{\lambda}\right)$ and standard elliptic theories that there exists some function $v$, such that $v_{\lambda} \rightarrow v$ in $C_{\text {loc }}^{2}(\Pi)$, where $\Pi$ is a half space of $\mathbb{R}^{3}$, and $v$ satisfies

$$
\begin{cases}-\Delta v=v^{5}, \quad|v| \leqslant 1 & \text { in } \Pi, \\ v=0 & \text { on } \partial \Pi, \\ v(0)=1, \quad \nabla v(0)=0, & \\ \|v\|^{2} \leqslant 2 S^{3 / 2}, \quad\|v\|_{L^{6}} \leqslant c . & \end{cases}
$$

But if $\Pi$ is a half space of $\mathbb{R}^{3}$, by Pohozaev Identity, $v$ has to vanish identically. Thus, we derive a contradiction and our lemma follows.

From Lemma 3.4, we derive that there exists some function $v$, such that, $v_{\lambda} \rightarrow v$ in $C_{\text {loc }}^{2}\left(\mathbb{R}^{3}\right)$, and $v$ satisfies

$$
\left\{\begin{array}{l}
-\Delta v=v^{5}, \quad|v| \leqslant 1 \quad \text { in } \mathbb{R}^{3}, \\
v(0)=1, \quad \nabla v(0)=0, \\
\|v\|^{2} \leqslant 2 S^{3 / 2}, \quad\|v\|_{L^{6}} \leqslant c .
\end{array}\right.
$$

But, we know that if $v$ is a sign changing solution, then $\|v\|^{2}>2 S^{3 / 2}$ (see p. 170 of [17]). Then $v$ has to be positive and therefore, it follows from [9] that

$$
v(y)=\delta_{\left(0, \alpha_{0}\right)}(y), \quad \text { with } \alpha_{0}=1 / \sqrt{3} .
$$

Thus

$$
M_{\lambda,+}^{-1} u_{\lambda}\left(a_{\lambda, 1}+M_{\lambda,+}^{-2} y\right)-\delta_{\left(0, \alpha_{0}\right)}(y) \rightarrow 0 \quad \text { in } C_{\mathrm{loc}}^{2}\left(\mathbb{R}^{3}\right) \text { as } \lambda \rightarrow \bar{\lambda}(\Omega) .
$$


Observe that

$$
M_{\lambda,+}^{-1} u_{\lambda}\left(a_{\lambda, 1}+M_{\lambda,+}^{-2} y\right)-\delta_{\left(0, \alpha_{0}\right)}(y)=M_{\lambda,+}^{-1}\left(u_{\lambda}(x)-\delta_{\left(a_{\lambda, 1}, \mu_{\lambda, 1}\right)}(x)\right),
$$

where $\mu_{\lambda, 1}=3^{-1 / 2} M_{\lambda,+}^{2}$.

Lemma 3.5. Let $u_{\lambda, 1}(x)=u_{\lambda}(x)-P \delta_{\left(a_{\lambda, 1}, \mu_{\lambda, 1}\right)}(x)$. Then we have
(i) $\int_{\Omega}\left|\nabla u_{\lambda, 1}\right|^{2}=\int_{\Omega}\left|\nabla u_{\lambda}\right|^{2}-S^{3 / 2}+\mathrm{o}(1) \quad$ as $\lambda \rightarrow \bar{\lambda}(\Omega)$,
(ii) $\int_{\Omega}\left|u_{\lambda, 1}\right|^{6}=\int_{\Omega}\left|u_{\lambda}\right|^{6}-S^{3 / 2}+\mathrm{o}(1) \quad$ as $\lambda \rightarrow \bar{\lambda}(\Omega)$.

Proof. We have

$$
\int_{\Omega}\left|\nabla u_{\lambda, 1}\right|^{2}=\int_{\Omega}\left|\nabla u_{\lambda}\right|^{2}+\int_{\Omega}\left|\nabla P \delta_{\left(a_{\lambda, 1}, \mu_{\lambda, 1}\right)}\right|^{2}-2 \int_{\Omega} \nabla u_{\lambda} \nabla P \delta_{\left(a_{\lambda, 1}, \mu_{\lambda, 1}\right)} .
$$

According to Bahri [4] (see also Rey [14]), we have thanks to Lemma 3.4

$$
\int_{\Omega}\left|\nabla P \delta_{\left(a_{\lambda, 1}, \mu_{\lambda, 1}\right)}\right|^{2}=S^{3 / 2}+\mathrm{o}(1) \quad \text { as } \lambda \rightarrow \bar{\lambda}(\Omega) .
$$

We also have

$$
\begin{aligned}
\int_{\Omega} \nabla u_{\lambda} \nabla P \delta_{\left(a_{\lambda, 1}, \mu_{\lambda, 1}\right)} & =\int_{\Omega} u_{\lambda} \delta_{\left(a_{\lambda, 1}, \mu_{\lambda, 1}\right)}^{5}=\int_{\Omega}\left(u_{\lambda}-\delta_{\left(a_{\lambda, 1}, \mu_{\lambda, 1}\right)}\right) \delta_{\left(a_{\lambda, 1}, \mu_{\lambda, 1}\right)}^{5}+\int_{\Omega} \delta_{\left(a_{\lambda, 1}, \mu_{\lambda, 1}\right)}^{6} \\
& =\int_{\Omega_{\lambda}}\left(v_{\lambda}-\delta_{\left(0, \alpha_{0}\right)}\right) \delta_{\left(0, \alpha_{0}\right)}^{5}+\int_{\Omega} \delta_{\left(a_{\lambda, 1}, \mu_{\lambda, 1}\right)}^{6} .
\end{aligned}
$$

Using Lemma 3.4 and Bahri [4] (see also Rey [14]), we have

$$
\int_{\Omega} \delta_{\left(a_{\lambda, 1}, \mu_{\lambda, 1}\right)}^{6}=S^{3 / 2}+\mathrm{o}(1) \quad \text { as } \lambda \rightarrow \bar{\lambda}(\Omega) .
$$

We now notice that

$$
\int_{\mathbb{R}^{3} \backslash B(0, R)}\left(v_{\lambda}-\delta_{\left(0, \alpha_{0}\right)}\right) \delta_{\left(0, \alpha_{0}\right)}^{5}=\mathrm{o}(1), \quad \text { for } R \text { large enough, }
$$

and

$$
\int_{B(0, R)}\left(v_{\lambda}-\delta_{\left(0, \alpha_{0}\right)}\right) \delta_{\left(0, \alpha_{0}\right)}^{5}=\mathrm{o}(1), \quad \text { because of } v_{\lambda} \rightarrow \delta_{\left(0, \alpha_{0}\right)} \text { in } C_{\text {loc }}^{2}\left(\mathbb{R}^{3}\right) .
$$

Then

$$
\int_{\Omega} \nabla u_{\lambda} \nabla P \delta_{\left(a_{\lambda, 1}, \mu_{\lambda, 1}\right)}=S^{3 / 2}+\mathrm{o}(1) .
$$

Therefore claim (i) follows from (3.6), (3.7) and (3.8). The proof of claim (ii) is similar to the proof of claim (i), so we will omit it.

Now, let us introduce the following notations: 


$$
\begin{aligned}
& h_{\lambda}:=\max _{x \in \Omega}\left(\left|x-a_{\lambda, 1}\right|^{1 / 2}\left|u_{\lambda}(x)\right|\right), \\
& Z_{\lambda}:=\left\{x \in \Omega \mid u_{\lambda}(x)=0\right\}, \\
& \Omega_{\lambda,+}:=\text { the connected component of } \Omega \backslash Z_{\lambda} \text { which contains } a_{\lambda, 1}, \\
& d_{\lambda, 1}:=d\left(a_{\lambda, 1}, \partial \Omega_{\lambda,+}\right) .
\end{aligned}
$$

Proposition 3.6. We have that

$$
h_{\lambda}:=\max _{x \in \Omega}\left(\left|x-a_{\lambda, 1}\right|^{1 / 2}\left|u_{\lambda}(x)\right|\right) \rightarrow+\infty \quad \text { as } \lambda \rightarrow \bar{\lambda}(\Omega) .
$$

Proof. First, we recall that the function $v_{\lambda}$, defined in (3.4), converges to $\delta_{\left(0, \alpha_{0}\right)}$ in $C_{\text {loc }}^{2}\left(\mathbb{R}^{3}\right)$. Therefore $d_{\lambda, 1} M_{\lambda,+}^{2} \rightarrow$ $+\infty$ as $\lambda \rightarrow \bar{\lambda}(\Omega)$. Secondly, we are going to prove the following crucial claim:

$$
h_{\lambda} \leqslant c \Longrightarrow d_{\lambda, 1} \nrightarrow 0 \quad \text { as } \lambda \rightarrow \bar{\lambda}(\Omega),
$$

where $c$ is a positive constant independent of $\lambda$, for $\lambda$ close to $\bar{\lambda}(\Omega)$.

Arguing by contradiction, we assume that $h_{\lambda} \leqslant c$ and $d_{\lambda, 1} \rightarrow 0$ as $\lambda \rightarrow \bar{\lambda}(\Omega)$. We set

$$
w_{\lambda}(y)=d_{\lambda, 1}^{1 / 2} u_{\lambda}\left(a_{\lambda, 1}+d_{\lambda, 1} y\right), \quad y \in \widetilde{\Omega}_{\lambda,+}:=d_{\lambda, 1}^{-1}\left(\Omega_{\lambda,+}-a_{\lambda, 1}\right) .
$$

Observe that $B(0,1) \subset \widetilde{\Omega}_{\lambda,+}$, and we have

$$
\left\{\begin{array}{l}
|y|^{1 / 2} w_{\lambda}(y) \leqslant c, \quad \text { for all } y \in B(0,1), \\
w_{\lambda}(0)=d_{\lambda, 1}^{1 / 2} u_{\lambda}\left(a_{\lambda, 1}\right)=d_{\lambda, 1}^{1 / 2} M_{\lambda,+} \rightarrow+\infty \quad \text { as } \lambda \rightarrow \bar{\lambda}(\Omega) .
\end{array}\right.
$$

Therefore 0 is an isolated blow-up point of $w_{\lambda}$ (see Appendix A for definition). Notice that $w_{\lambda}$ satisfies

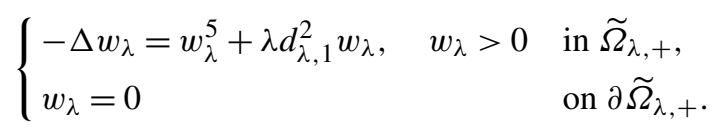

Then it follows from Proposition A.9 in Appendix A that 0 is an isolated simple blow-up point in $B(0,1)$. By Propositions A.6 and A.7 of Appendix A, we know that there exist positive constants $c_{1}$ and $c_{2}$ such that

$$
c_{1} w_{\lambda}(0)\left(1+\alpha_{0}^{2} w_{\lambda}^{4}(0)|y|^{2}\right)^{-1 / 2} \leqslant w_{\lambda}(y) \leqslant c_{2} w_{\lambda}^{-1}(0)|y|^{-1} \quad \text { for } y \in B(0,1 / 2) \backslash\{0\} .
$$

Observe that

$$
\Delta w_{\lambda}+V_{\lambda} w_{\lambda}=0, \quad \text { with } V_{\lambda}=w_{\lambda}^{4}+\lambda d_{\lambda, 1}^{2} .
$$

Notice that, since $h_{\lambda}$ is bounded, we have

$$
V_{\lambda}(y) \leqslant c \quad \text { for all } y \in \widetilde{\Omega}_{\lambda,+} \backslash B(0,1 / 4) .
$$

By Harnack Inequality (see Corollary 8.21 of [12]), we deduce that, for any compact set $K$ of $\widetilde{\Omega}_{\lambda,+} \backslash\{0\}$, we have

$$
w_{\lambda}(y) \leqslant c_{K} w_{\lambda}(0)^{-1} \text { for all } y \in K .
$$

Now, we set

$$
\widetilde{w}_{\lambda}(y)=w_{\lambda}(0) w_{\lambda}(y) .
$$

It is easy to see that $\widetilde{w}_{\lambda}$ satisfies

$$
\left\{\begin{array}{lll}
-\Delta \widetilde{w}_{\lambda}=\widetilde{w}_{\lambda}(0)^{-4} \widetilde{w}_{\lambda}^{5}+\lambda d_{\lambda, 1}^{2} \widetilde{w}_{\lambda}, & \widetilde{w}_{\lambda}>0 & \text { in } \widetilde{\Omega}_{\lambda,+}, \\
\widetilde{w}_{\lambda}=0 & \text { on } \partial \widetilde{\Omega}_{\lambda,+} .
\end{array}\right.
$$

By (3.17), $\widetilde{w}_{\lambda}$ is bounded in any compact set of $\widetilde{\Omega}_{\lambda,+} \backslash\{0\}$. It follows from standard elliptic theories that $\widetilde{w}_{\lambda}$ converges in $C_{\text {loc }}^{2}(\Pi \backslash\{0\})$ to some positive function $\widetilde{w} \in C^{2}(\Pi \backslash\{0\})$, where $\Pi$ is the limit domain of $\widetilde{\Omega}_{\lambda,}+$ when $\lambda \rightarrow \bar{\lambda}(\Omega)$. Since $d_{\lambda, 1} \rightarrow 0$, we see that $\widetilde{w}$ satisfies 


$$
\begin{cases}-\Delta \widetilde{w}=0, \quad \widetilde{w} \geqslant 0 & \text { in } \Pi \backslash\{0\}, \\ \widetilde{w}=0 & \text { on } \partial \Pi .\end{cases}
$$

It follows from (3.16) that 0 is a nonremovable singularity of $\widetilde{w}$. Therefore

$$
\widetilde{w}_{\lambda} \rightarrow \alpha G_{\Pi}(0, \cdot) \quad \text { in } C_{\text {loc }}^{2}(\Pi \backslash\{0\}),
$$

where $G_{\Pi}(0, \cdot)$ is the Green function of the Laplacian operator with Dirichlet boundary condition defined on the limit domain and $\alpha$ is a positive constant. Such a Green function can be written as

$$
G_{\Pi}(0, y)=|y|^{-1}-H(0, y),
$$

where by the maximum principle $H(0, y)>0$.

Applying Pohozaev Identity in the form of Theorem 1.1 of [13], we derive that, for $0<r<1 / 2$

$$
\lambda d_{\lambda, 1}^{2} \int_{B_{r}} w_{\lambda}^{2}-\frac{r}{6} \int_{\partial B_{r}} w_{\lambda}^{6}-\frac{r}{2} \lambda d_{\lambda, 1}^{2} \int_{\partial B_{r}} w_{\lambda}^{2}=\int_{\partial B_{r}} B\left(r, x, w_{\lambda}, \nabla w_{\lambda}\right) \mathrm{d} x,
$$

where $B_{r}=B(0, r)$ and where

$$
B(r, x, u, \nabla u)=\frac{1}{2} u \frac{\partial u}{\partial v}-\frac{r}{2}|\nabla u|^{2}+r\left(\frac{\partial u}{\partial v}\right)^{2} .
$$

Multiplying (3.18) by $w_{\lambda}^{2}(0)$ and using the homogeneity of the operator $B$, we obtain

$$
\lambda d_{\lambda, 1}^{2} \int_{B_{r}} \widetilde{w}_{\lambda}^{2}-\frac{r}{6} w_{\lambda}^{-4}(0) \int_{\partial B_{r}} \widetilde{w}_{\lambda}^{6}-\frac{r}{2} \lambda d_{\lambda, 1}^{2} \int_{\partial B_{r}} \widetilde{w}_{\lambda}^{2}=\int_{\partial B_{r}} B\left(r, x, \widetilde{w}_{\lambda}, \nabla \widetilde{w}_{\lambda}\right) \mathrm{d} x .
$$

Using (3.16), we derive that

$$
\begin{aligned}
& r w_{\lambda}^{-4}(0) \int_{\partial B_{r}} \widetilde{w}_{\lambda}^{6} \leqslant c w_{\lambda}^{-4}(0) r^{-3} \rightarrow 0 \quad \text { as } \lambda \rightarrow \bar{\lambda}(\Omega), \\
& \lambda d_{\lambda, 1}^{2} \int_{B_{r}} \widetilde{w}_{\lambda}^{2} \leqslant c \lambda d_{\lambda, 1}^{2} \int_{B_{r}} \frac{\mathrm{d} y}{|y|^{2}} \leqslant c \lambda d_{\lambda, 1}^{2} \rightarrow 0 \quad \text { as } \lambda \rightarrow \bar{\lambda}(\Omega), \\
& \lambda d_{\lambda, 1}^{2} \int_{\partial B_{r}} \widetilde{w}_{\lambda}^{2} \leqslant c \lambda d_{\lambda, 1}^{2} \rightarrow 0 \quad \text { as } \lambda \rightarrow \bar{\lambda}(\Omega) .
\end{aligned}
$$

Therefore the left-hand side of (3.20) tends to zero as $\lambda \rightarrow \bar{\lambda}(\Omega)$.

Now, since

$$
\widetilde{w}_{\lambda} \rightarrow \alpha G_{\Pi}(0, \cdot) \quad \text { in } C_{\text {loc }}^{2}\left(\partial B_{r}\right) \text { for } 0<r<1 / 2
$$

and for $r$ small

$$
G_{\Pi}(0, x)=|x|^{-1}-H(0,0)+\mathrm{o}(|x|), \quad \text { with }|x|=r,
$$

we deduce that

$$
\lim _{\lambda \rightarrow \bar{\lambda}(\Omega), r \rightarrow 0} \int_{\partial B_{r}} B\left(r, x, \widetilde{w}_{\lambda}, \nabla \widetilde{w}_{\lambda}\right) \mathrm{d} x=\frac{1}{2} \alpha^{2} \omega_{3} H(0,0)>0,
$$

where $\omega_{3}$ is the area of the unit sphere in $\mathbb{R}^{3}$. This leads a contradiction and therefore claim (3.13) follows.

Now, we are going to prove Proposition 3.6. Arguing by contradiction, we suppose that

$$
h_{\lambda} \leqslant c, \quad \text { with } c \text { is a positive constant independent of } \lambda \text {. }
$$

By claim (3.13), we have $d_{\lambda, 1} \geqslant c>0$ as $\lambda \rightarrow \bar{\lambda}(\Omega)$. Observe that $\left|a_{\lambda, 1}-x_{\lambda,-}\right| \geqslant d_{\lambda, 1}$, where $x_{\lambda,-} \in \Omega$ such that $\left|u_{\lambda}\left(x_{\lambda,-}\right)\right|=\max u_{\lambda}^{-}$. Thus, using Lemma 3.3, we obtain

$$
\left|a_{\lambda, 1}-x_{\lambda,-}\right|^{1 / 2}\left|u_{\lambda}\left(x_{\lambda,-}\right)\right| \rightarrow+\infty \quad \text { as } \lambda \rightarrow \bar{\lambda}(\Omega) .
$$


This implies that $h_{\lambda} \rightarrow+\infty$ as $\lambda \rightarrow \bar{\lambda}(\Omega)$. Therefore we obtain a contradiction and our proposition follows.

Now, let $x_{\lambda, 2} \in \Omega$ and $M_{\lambda, 2}>0$ such that

$$
h_{\lambda}=\left|a_{\lambda, 1}-x_{\lambda, 2}\right|^{1 / 2} M_{\lambda, 2},
$$

where $h_{\lambda}$ is defined by (3.9). We set

$$
\tilde{u}_{\lambda}(y)=M_{\lambda, 2}^{-1} u_{\lambda}\left(x_{\lambda, 2}+M_{\lambda, 2}^{-2} y\right) \quad \text { for } y \in \Omega_{\lambda, 2}:=M_{\lambda, 2}^{2}\left(\Omega-x_{\lambda, 2}\right) .
$$

Recall that, for any $x \in \Omega$, we have

$$
\left|x-a_{\lambda, 1}\right|^{1 / 2}\left|u_{\lambda}(x)\right| \leqslant\left|x_{\lambda, 2}-a_{\lambda, 1}\right|^{1 / 2}\left|u_{\lambda}\left(x_{\lambda, 2}\right)\right| .
$$

Notice that, for $x \in B\left(x_{\lambda, 2},\left|x_{\lambda, 2}-a_{\lambda, 1}\right| / 2\right)$, we have $\left|x-a_{\lambda, 1}\right| \geqslant\left|x_{\lambda, 2}-a_{\lambda, 1}\right| / 2$. Hence

$$
M_{\lambda, 2}^{-1}\left|u_{\lambda}(x)\right| \leqslant \sqrt{2} \quad \text { for any } x \in B\left(x_{\lambda, 2},\left|x_{\lambda, 2}-a_{\lambda, 1}\right| / 2\right) \cap \Omega .
$$

Thus, we obtain

$$
\left|\tilde{u}_{\lambda}(y)\right| \leqslant \sqrt{2} \text { for any } y \in B\left(0, M_{\lambda, 2}^{2}\left|x_{\lambda, 2}-a_{\lambda, 1}\right| / 2\right) \cap \Omega_{\lambda, 2} .
$$

As in Lemma 3.4, we can prove that

$$
M_{\lambda, 2}^{2} d\left(x_{\lambda, 2}, \partial \Omega\right) \rightarrow+\infty \quad \text { as } \lambda \rightarrow \bar{\lambda}(\Omega),
$$

and therefore $\tilde{u}_{\lambda}$ converges in $C_{\text {loc }}^{2}\left(\mathbb{R}^{3}\right)$ to some function $\varphi$ such that

$$
\left\{\begin{array}{l}
-\Delta \varphi=\varphi^{5} \quad \text { in } \mathbb{R}^{3} \\
|\varphi(0)|=1, \quad|\varphi(x)| \leqslant \sqrt{2}, \\
\|\varphi\|^{2} \leqslant 2 S^{3 / 2}
\end{array}\right.
$$

Thus $\varphi$ does not change sign and therefore two cases may occur:

Case 1: $u_{\lambda}\left(x_{\lambda, 2}\right)>0$. In this case $\varphi>0$ and therefore there exist $b \in \mathbb{R}^{3}$ and $\mu>0$ such that $\varphi=\delta_{(b, \mu)}$. Using Proposition 3.6, we see that

$$
\left\|u_{\lambda}^{+}\right\|^{2} \geqslant 2 S^{3 / 2}+\mathrm{o}(1) \quad \text { as } \lambda \rightarrow \bar{\lambda}(\Omega)
$$

which contradicts Lemma 3.2. Thus this case cannot happen.

Case 2: $u_{\lambda}\left(x_{\lambda, 2}\right)<0$. Thus $\varphi<0$ and therefore there exist $b \in \mathbb{R}^{3}$ and $\mu>0$ such that $\varphi=-\delta_{(b, \mu)}$. Since $b$ is a nondegenerate critical point of $\delta_{(b, \mu)}$, there exists $b_{\lambda} \rightarrow b$ as $\lambda \rightarrow \bar{\lambda}(\Omega)$, such that $\nabla \tilde{u}_{\lambda}\left(b_{\lambda}\right)=0$ and $\tilde{u}_{\lambda}\left(b_{\lambda}\right) \rightarrow$ $3^{1 / 4} \mu^{1 / 2}$. We see that

$$
\tilde{u}_{\lambda}+\delta_{\left(b_{\lambda}, 3^{-1 / 2} \tilde{u}_{\lambda}\left(b_{\lambda}\right)^{2}\right)} \rightarrow 0 \quad \text { in } C_{\text {loc }}^{2}\left(\mathbb{R}^{3}\right) \text { as } \lambda \rightarrow \bar{\lambda}(\Omega) .
$$

Thus we have found a second blow-up point $a_{\lambda, 2}$ of $u_{\lambda}$ with the concentration $\mu_{\lambda, 2}$ defined by

$$
a_{\lambda, 2}=x_{\lambda, 2}+M_{\lambda, 2}^{-1} b_{\lambda} \quad \text { and } \quad \mu_{\lambda, 2}=3^{-1 / 2} u_{\lambda}\left(a_{\lambda, 2}\right)^{2} .
$$

Clearly, we have

$$
\nabla u_{\lambda}\left(a_{\lambda, 2}\right)=0 \quad \text { and } \quad \int_{\Omega} \nabla P \delta_{\left(a_{\lambda, 1}, \mu_{\lambda, 1}\right)} \cdot \nabla P \delta_{\left(a_{\lambda, 2}, \mu_{\lambda, 2}\right)} \rightarrow 0 \quad \text { as } \lambda \rightarrow \bar{\lambda}(\Omega) .
$$

Therefore, as in Lemma 3.5, we obtain

$$
\left\|u_{\lambda}-P \delta_{\left(a_{\lambda, 1}, \mu_{\lambda, 1}\right)}+P \delta_{\left(a_{\lambda, 2}, \mu_{\lambda, 2}\right)}\right\| \rightarrow 0 \quad \text { as } \lambda \rightarrow \bar{\lambda}(\Omega) .
$$

Now, we are in position to prove Theorem 3.1.

Proof of Theorem 3.1. We have already proved the existence of two local extremum points $a_{\lambda, 1}, a_{\lambda, 2}$ of $u_{\lambda}$ satisfying (3.21) and

$$
\mu_{\lambda, i}:=3^{-1 / 2} u_{\lambda}\left(a_{\lambda, i}\right)^{2} \rightarrow+\infty, \quad \mu_{\lambda, i} d\left(a_{\lambda, i}, \partial \Omega\right) \rightarrow+\infty \quad \text { as } \lambda \rightarrow \bar{\lambda}(\Omega), \text { for } i \in\{1,2\} .
$$


It remains to prove that there exists $\alpha>0$ such that

$$
d\left(a_{\lambda, i}, \partial \Omega\right) \geqslant \alpha, \quad \text { for } i \in\{1,2\}, \quad \text { and } \quad\left|a_{\lambda, 1}-a_{\lambda, 2}\right| \geqslant \alpha, \quad \text { as } \lambda \rightarrow \bar{\lambda}(\Omega) .
$$

Let $\mathcal{S}=\left\{a_{\lambda, 1}, a_{\lambda, 2}\right\}$. We first prove that there exists $c>0$ such that

$$
h_{\lambda, 2}:=\max _{\Omega}\left(d(x, \mathcal{S})^{1 / 2}\left|u_{\lambda}(x)\right|\right) \leqslant c \quad \text { for } \lambda \text { close to } \bar{\lambda}(\Omega) .
$$

Arguing by contradiction, we assume that

$$
h_{\lambda, 2} \rightarrow+\infty \quad \text { as } \lambda \rightarrow \bar{\lambda}(\Omega)
$$

Thus, as before, we can build a new blow-up point which ignores $a_{\lambda, 1}$ and $a_{\lambda, 2}$, that is, the image under the new scaling of $a_{\lambda, 1}$ and $a_{\lambda, 2}$, will escape to infinity when $\lambda$ tends to $\bar{\lambda}(\Omega)$. Clearly, this implies that

$$
\left\|u_{\lambda}\right\|^{2} \geqslant 3 S^{3 / 2}+o(1) \quad \text { as } \lambda \rightarrow \bar{\lambda}(\Omega)
$$

which contradicts the assumption of Theorem 3.1. Hence (3.23) follows.

Now, let

$$
\Omega_{\lambda,-} \text { be the connected component of } \Omega \backslash Z_{\lambda} \text { which contains } a_{\lambda, 2},
$$

where $Z_{\lambda}$ is defined by (3.10). Set

$$
d_{\lambda, 2}:=d\left(a_{\lambda, 2}, \partial \Omega_{\lambda,-}\right) .
$$

We distinguish two cases:

Case 1: $d_{\lambda, 1} \leqslant d_{\lambda, 2}$. In this case, let us prove that

$$
d_{\lambda, 1} \nrightarrow 0 \quad \text { as } \lambda \rightarrow \bar{\lambda}(\Omega) .
$$

Arguing by contradiction, we assume that $d_{\lambda, 1} \rightarrow 0$ as $\lambda \rightarrow \bar{\lambda}(\Omega)$. Since $h_{\lambda, 2}$ is bounded, 0 is an isolated blow-up point of the function $w_{\lambda}$, defined in (3.14), and thus arguing as in the proof of Proposition 3.6, we derive a contradiction.

Case 2: $d_{\lambda, 2} \leqslant d_{\lambda, 1}$. As in case 1 , we prove that $d_{\lambda, 2} \nrightarrow 0$ as $\lambda \rightarrow \bar{\lambda}(\Omega)$.

Now, since $d\left(a_{\lambda, i}, \partial \Omega\right) \geqslant d_{\lambda, i}$, for $i \in\{1,2\}$ and $\left|a_{\lambda, 1}-a_{\lambda, 2}\right| \geqslant d_{\lambda, 1}+d_{\lambda, 2}$, we deduce (3.22) and therefore our theorem is proved.

\section{Study of concentration speeds}

Here we prove that the speeds of the two blow-up points $a_{\lambda, 1}$ and $a_{\lambda, 2}$, constructed in Theorem 3.1, are of the same order.

Theorem 4.1. Under the assumptions of Theorem 3.1, there exists a constant $\bar{c}>0$ such that

$$
\frac{1}{\bar{c}} \leqslant-\frac{u_{\lambda}\left(a_{\lambda, 1}\right)}{u_{\lambda}\left(a_{\lambda, 2}\right)} \leqslant \bar{c} \quad \text { for } \lambda \text { close to } \bar{\lambda}(\Omega),
$$

where $a_{\lambda, 1}$ and $a_{\lambda, 2}$ are the blow-up points defined in Theorem 3.1 .

Proof. By Theorem 3.1, we know that $\bar{a}_{1}=\lim _{\lambda \rightarrow \bar{\lambda}(\Omega)} a_{\lambda, 1}$, and $\bar{a}_{2}=\lim _{\lambda \rightarrow \bar{\lambda}(\Omega)} a_{\lambda, 2}$ are isolated simple blow-up points of $\left(u_{\lambda}\right)$. Thus, there exist $r_{0}>0, c_{1}>0$ and $c_{2}>0$ such that

$$
\begin{aligned}
& 0<\frac{c_{1} u_{\lambda}\left(a_{\lambda, 1}\right)}{\left(1+\alpha_{0}^{2} u_{\lambda}^{4}\left(a_{\lambda, 1}\right)\left|x-a_{\lambda, 1}\right|^{2}\right)^{1 / 2}} \leqslant u_{\lambda}(x) \leqslant \frac{c_{2} u_{\lambda}^{-1}\left(a_{\lambda, 1}\right)}{\left|x-a_{\lambda, 1}\right|}, \quad \forall x \in B\left(a_{\lambda, 1}, r_{0}\right) \backslash\left\{a_{\lambda, 1}\right\}, \\
& \frac{c_{2} u_{\lambda}^{-1}\left(a_{\lambda, 2}\right)}{\left|x-a_{\lambda, 2}\right|} \leqslant u_{\lambda}(x) \leqslant \frac{c_{1} u_{\lambda}\left(a_{\lambda, 2}\right)}{\left(1+\alpha_{0}^{2} u_{\lambda}^{4}\left(a_{\lambda, 2}\right)\left|x-a_{\lambda, 2}\right|^{2}\right)^{1 / 2}}<0, \quad \forall x \in B\left(a_{\lambda, 2}, r_{0}\right) \backslash\left\{a_{\lambda, 2}\right\},
\end{aligned}
$$

where $\alpha_{0}=1 / \sqrt{3}$.

We also know that (see Section 3)

$$
h_{\lambda, 2}:=\max _{x \in \Omega}\left(d(x, \mathcal{S})^{1 / 2}\left|u_{\lambda}(x)\right|\right) \leqslant c \quad \text { for } \lambda \text { close to } \bar{\lambda}(\Omega),
$$


where $\mathcal{S}=\left\{a_{\lambda, 1}, a_{\lambda, 2}\right\}$.

Thus, we derive that $u_{\lambda}$ is bounded in each compact set $K$ of $\Omega \backslash \mathcal{S}$. We now consider

$$
L:=\left(-\Delta-\bar{V}_{\lambda}\right), \quad \text { with } \bar{V}_{\lambda}=\left(\left|u_{\lambda}\right|^{4}+\lambda\right) I d .
$$

We know that $\bar{V}_{\lambda}$ is bounded in each compact set of $\Omega \backslash\left\{a_{\lambda, 1}, a_{\lambda, 2}\right\}$ and $L u_{\lambda}=0$. Thus for any compact set $K_{+}$of $\Omega_{\lambda,+} \backslash\left\{a_{\lambda, 1}\right\}$, we have by (4.1) and Harnack Inequality

$$
0<u_{\lambda}(x) \leqslant C_{K_{+}} u_{\lambda}^{-1}\left(a_{\lambda, 1}\right) \quad \forall x \in K_{+},
$$

where $\Omega_{\lambda,+}$ is defined by (3.11) and $C_{K_{+}}$is a positive constant. In the same way, we have for any compact set $K_{-}$of $\Omega_{\lambda,-} \backslash\left\{a_{\lambda, 2}\right\}$

$$
C_{K_{-}} u_{\lambda}^{-1}\left(a_{\lambda, 2}\right) \leqslant u_{\lambda}(x)<0 \quad \forall x \in K_{-},
$$

where $\Omega_{\lambda,-}$ is defined by (3.24) and $C_{K_{-}}$is a positive constant.

Now we need to estimate $u_{\lambda}$ in $D_{ \pm}:=\left\{x \in \Omega_{\lambda, \pm} \mid d\left(x, \partial \Omega_{\lambda, \pm}\right)<d_{0}\right\}$ where $d_{0}$ is a fixed positive constant. For this fact, observe that $u_{\lambda}$ is bounded in $\Omega_{0}:=\Omega \backslash \bigcup B\left(a_{\lambda, i}, r_{0} / 2\right)$ which implies that $\Delta u_{\lambda}$ is bounded in $\Omega_{0}$. Thus, since $u_{\lambda}=0$ on $\partial \Omega_{\lambda, \pm}$, we derive that $\left|\nabla u_{\lambda}\right| \leqslant c$ in $D_{ \pm}$and therefore $\left|u_{\lambda}\right| \leqslant c d_{0}$ in $D_{ \pm}$, where $c$ is a constant independent of $\lambda$. Using also (4.3), (4.4) for $K_{ \pm}:=\Omega_{\lambda, \pm} \backslash\left(D_{ \pm} \cup B\left(a_{\lambda, \pm}, r_{0} / 2\right)\right)$ and Corollary 2.2, we can choose $d_{0}$ such that

$$
\left|u_{\lambda}\right|_{L^{\infty}\left(\Omega_{0}\right)}^{4}+\lambda \leqslant c^{\prime}<\lambda_{1}(\Omega) \text { for } \lambda \text { close to } \bar{\lambda}(\Omega),
$$

where $c^{\prime}$ is a positive constant independent of $\lambda$ and where we have used the fact that $\bar{\lambda}(\Omega)<\lambda_{1}(\Omega)$.

Therefore the operator $L$ satisfies the maximum principle in $\Omega_{0}$. We deduce that

$$
-\sup _{\partial \Omega_{0}} u_{\lambda}^{-} \leqslant u_{\lambda}(x) \leqslant \sup _{\partial \Omega_{0}} u_{\lambda}^{+} \quad \forall x \in \Omega_{0} .
$$

Hence, using (4.1) and (4.2), we obtain

$$
c_{1}^{\prime} u_{\lambda}^{-1}\left(a_{\lambda, 2}\right) \leqslant u_{\lambda}(x) \leqslant c_{2}^{\prime} u_{\lambda}^{-1}\left(a_{\lambda, 1}\right) \quad \forall x \in \Omega_{0},
$$

where $c_{1}^{\prime}$ and $c_{2}^{\prime}$ are positive constants independent of $\lambda$ and $\Omega_{0}:=\Omega \backslash \bigcup B\left(a_{\lambda, i}, r_{0} / 2\right)$.

To prove Theorem 4.1, we argue by contradiction and we will prove that the ratio $-\frac{u_{\lambda}\left(a_{\lambda, 1}\right)}{u_{\lambda}\left(a_{\lambda, 2}\right)}$ cannot tend to zero nor to $+\infty$ when $\lambda \rightarrow \bar{\lambda}(\Omega)$.

Step 1: $-\frac{u_{\lambda}\left(a_{\lambda, 1}\right)}{u_{\lambda}\left(a_{\lambda, 2}\right)} \rightarrow 0$ cannot occur. Assume that it tends to zero when $\lambda \rightarrow \bar{\lambda}(\Omega)$. By (4.1), (4.2) and (4.5), we have

$$
\left\{\begin{array}{l}
c_{1}^{\prime} \frac{u_{\lambda}\left(a_{\lambda, 1}\right)}{u_{\lambda}\left(a_{\lambda, 2}\right)} \leqslant u_{\lambda}\left(a_{\lambda, 1}\right) u_{\lambda}(x) \leqslant c_{2}^{\prime} \quad \forall x \in \Omega_{0}, \\
\frac{c_{1} u_{\lambda}^{2}\left(a_{\lambda, 1}\right)}{\left(1+\alpha_{0}^{2} u_{\lambda}^{4}\left(a_{\lambda, 1}\right)\left|x-a_{\lambda, 1}\right|^{2}\right)^{1 / 2}} \leqslant u_{\lambda}\left(a_{\lambda, 1}\right) u_{\lambda}(x) \leqslant \frac{c_{2}}{\left|x-a_{\lambda, 1}\right|} \quad \forall x \in B\left(a_{\lambda, 1}, r_{0}\right) \backslash\left\{a_{\lambda, 1}\right\}, \\
\frac{c_{2} u_{\lambda}\left(a_{\lambda, 1}\right)}{\left|x-a_{\lambda, 2}\right| u_{\lambda}\left(a_{\lambda, 2}\right)} \leqslant u_{\lambda}\left(a_{\lambda, 1}\right) u_{\lambda}(x) \leqslant \frac{c_{1} u_{\lambda}\left(a_{\lambda, 1}\right) u_{\lambda}\left(a_{\lambda, 2}\right)}{\left(1+\alpha_{0}^{2} u_{\lambda}^{4}\left(a_{\lambda, 2}\right)\left|x-a_{\lambda, 2}\right|^{2}\right)^{1 / 2}} \quad \forall x \in B\left(a_{\lambda, 2}, r_{0}\right) \backslash\left\{a_{\lambda, 2}\right\} .
\end{array}\right.
$$

Thus, we derive that $W_{\lambda}:=u_{\lambda}\left(a_{\lambda, 1}\right) u_{\lambda}$ is bounded in each compact set $K \subset \Omega \backslash \mathcal{S}$. It follows from standard elliptic theories that $W_{\lambda} \rightarrow \bar{w}$ in $C_{\text {loc }}^{2}(\Omega \backslash \overline{\mathcal{S}})$, where $\overline{\mathcal{S}}=\left\{\bar{a}_{1}, \bar{a}_{2}\right\}$ is the limit set of the concentration points $a_{\lambda, 1}$ and $a_{\lambda, 2}$ and where $\bar{w}$ satisfies

$$
\begin{cases}-\Delta \bar{w}=\bar{\lambda}(\Omega) \bar{w}, \quad \bar{w} \geqslant 0 & \text { in } \Omega \backslash \overline{\mathcal{S}}, \\ \bar{w}=0 & \text { on } \partial \Omega .\end{cases}
$$

From (4.6) and the fact that $-\frac{u_{\lambda}\left(a_{\lambda, 1}\right)}{u_{\lambda}\left(a_{\lambda, 2}\right)} \rightarrow 0$, it is easy to see that $\bar{a}_{1}$ is a nonremovable singularity of $\bar{w}$ but not $\bar{a}_{2}$. Thus

$$
\bar{w}(y)=\bar{\alpha} G_{\bar{\lambda}}\left(\bar{a}_{1}, y\right), \quad \bar{\alpha}>0,
$$

where $G_{\bar{\lambda}}$ is the Green function of $(-\Delta-\bar{\lambda}(\Omega))$ in $\Omega$ with Dirichlet boundary condition, that is, 


$$
\forall x \in \Omega \quad \begin{cases}(-\Delta-\bar{\lambda}(\Omega)) G_{\bar{\lambda}}(x, \cdot)=\omega_{3} \delta_{x} & \text { in } \Omega, \\ G_{\bar{\lambda}}(x, \cdot)=0 & \text { on } \partial \Omega,\end{cases}
$$

where $\omega_{3}$ is the area of the unit sphere of $\mathbb{R}^{3}$. Now, since $u_{\lambda}\left(a_{\lambda, 1}\right)>0$ and $u_{\lambda}\left(a_{\lambda, 2}\right)<0$, we see that $u_{\lambda}$ has to vanish at some point $x_{\lambda}$. Let $\bar{x}=\lim _{\lambda \rightarrow \bar{\lambda}(\Omega)} x_{\lambda}$. Then $\bar{x}$ belongs to some compact set of $\Omega \backslash \overline{\mathcal{S}}$ and therefore $\bar{w}(\bar{x})=0$ which contradicts (4.7) because of $G_{\bar{\lambda}}>0$ in $\Omega$, since we have assumed that $\bar{\lambda}(\Omega)<\lambda_{1}(\Omega)$. Step 1 is thus proved.

Step 2. $-\frac{u_{\lambda}\left(a_{\lambda, 2}\right)}{u_{\lambda}\left(a_{\lambda, 1}\right)} \rightarrow+\infty$ cannot occur. Step 2 can be proved in exactly the same way as Step 1, we just multiply by $u_{\lambda}\left(a_{\lambda, 2}\right)$ instead of $u_{\lambda}\left(a_{\lambda, 1}\right)$.

Next we are going to give an alternative proof of Corollary 1.2 in the case when $\bar{\lambda}(\Omega)$ is not achieved. Let us start by the following lemma:

Lemma 4.2. Assume that $\bar{\lambda}(\Omega)<\lambda_{1}(\Omega)$. If $\bar{\lambda}(\Omega)$ is not achieved and $u_{\lambda}$ is a family of sign changing solutions of $\left(\mathrm{P}_{\lambda}\right)$ which satisfies $\left\|u_{\lambda}\right\|_{\lambda}^{2} \leqslant 2 S^{3 / 2}$ with $\lambda \rightarrow \bar{\lambda}(\Omega)$, then

$$
\left\|u_{\lambda}\right\|^{2} \rightarrow 2 S^{3 / 2} \text { and } u_{\lambda} \rightarrow 0 \quad \text { in } H_{0}^{1}(\Omega) \text { as } \lambda \rightarrow \bar{\lambda}(\Omega) .
$$

Proof. Let $\bar{u}$ be such that $u_{\lambda} \rightarrow \bar{u}$ as $\lambda \rightarrow \bar{\lambda}(\Omega)$. Thus $\bar{u}$ is a solution of $\left(\mathrm{P}_{\bar{\lambda}(\Omega)}\right)$. Since $\bar{\lambda}(\Omega)$ is not achieved we derive that $\bar{u}$ does not change sign. We can assume that $\bar{u} \leqslant 0$.

We argue by contradiction and we assume that $\bar{u} \neq 0$. Thus by the maximum principle we deduce that $\bar{u}<0$. It is clear that the connected components of $u_{\lambda}^{+}$will degenerate as $\lambda \rightarrow \bar{\lambda}(\Omega)$.

It is easy to see that $u_{\lambda}^{+} \rightarrow 0$ and therefore as in the proof of Proposition 2.1 we derive that $\left\|u_{\lambda}^{+}\right\|^{2} \geqslant S^{3 / 2}(1+\mathrm{o}(1))$ and therefore, since $\left\|u_{\lambda}\right\|_{\lambda}^{2} \leqslant 2 S^{3 / 2}$, we obtain $\left\|u_{\lambda}^{-}\right\|_{\lambda}^{2} \leqslant S^{3 / 2}$.

Now let $M_{+}=\max \left|u_{\lambda}\right|=\left|u_{\lambda}\left(a_{+}\right)\right|$. It is easy to see that $M_{+} \rightarrow+\infty$ (if not, we obtain $\left|u_{\lambda}^{+}\right|_{L^{\infty}} \leqslant c$ and we have $u_{\lambda} \rightarrow 0$ thus $\left\|u_{\lambda}^{+}\right\| \rightarrow 0$ which is not true).

Thus we can prove that

$$
v_{\lambda}(X)=\frac{1}{M_{+}} u_{\lambda}\left(a_{+}+\frac{X}{M_{+}^{2}}\right), \quad \text { for } X \in M_{+}\left(\Omega-a_{+}\right)
$$

converges in $C_{\text {loc }}^{1}\left(\mathbb{R}^{3}\right)$ to $\operatorname{sign}\left(u_{\lambda}\left(a_{+}\right)\right) \delta_{\left(0, \alpha_{0}\right)}$. Furthermore, $u_{\lambda}\left(a_{+}\right)$has to be positive. Indeed, the function $\delta_{\left(0, \alpha_{0}\right)}$ cannot be in the description of $u_{\lambda}^{-}$(since $u_{\lambda}^{-} \rightarrow \bar{u}<0$ and $\left\|u_{\lambda}^{-}\right\|_{\lambda}^{2} \leqslant S^{3 / 2}$ ). Thus $a_{+}$is a concentration point with the speed $M_{+}^{2}$.

Now we introduce $h_{\lambda}$ as in (3.9). Two cases may occur.

1st case. $h_{\lambda} \rightarrow+\infty$. In this case, we can construct another concentration point $a_{2}$ with the speed $\lambda_{2}$ that means the function $\pm \delta_{\left(a_{2}, \lambda_{2}\right)}$ appears in the description of $u_{\lambda}$. Since we assumed that $\bar{u}<0$ we derive that $\left\|u_{\lambda}\right\|_{\lambda}^{2}$ is bigger than $2 S^{3 / 2}$ which is a contradiction with $\left\|u_{\lambda}\right\|_{\lambda}^{2} \leqslant 2 S^{3 / 2}$. Thus this case cannot occur.

2nd case. $h_{\lambda} \leqslant c$. In this case, we can use the claim (3.13) and we deduce that $d_{\lambda,+}:=d\left(a_{+}, \partial \Omega_{\lambda,+}\right) \nrightarrow 0$ where $\Omega_{\lambda,+}$ is defined by (3.11). (In the proof of this claim we used only the set $\Omega_{\lambda,+}$ thus the proof remains unchanged if $u_{\lambda}^{-} \rightarrow 0$ is not satisfied.)

Now it is easy to see that $\Omega_{\lambda,+}$ has to degenerate (because $u_{\lambda}$ converges weakly to a negative function in the whole domain) and therefore $d_{\lambda,+}$ has to tend to 0 which is a contradiction with the claim (3.13). Thus this case cannot occur neither.

The proof is thereby completed.

Proof of Corollary 1.2 when $\bar{\lambda}(\Omega)$ is not achieved. Let $G_{\bar{\lambda}}$ be the Green function of $(-\Delta-\bar{\lambda}(\Omega))$ defined by (4.8). Arguing by contradiction, we assume that $\bar{\lambda}(\Omega)<\lambda^{*}(\Omega)$. Thus $\left(\mathrm{P}_{\lambda}\right)$ has no positive solution $u_{\lambda}$ such that $\left\|u_{\lambda}\right\|_{\lambda}^{2} \leqslant$ $S^{3 / 2}$, where $\left\|u_{\lambda}\right\|_{\lambda}^{2}=\int_{\Omega}\left|\nabla u_{\lambda}\right|^{2}-\lambda \int_{\Omega} u_{\lambda}^{2}$. Therefore, using the result of [11], we derive that

$$
\forall x \in \Omega \quad H_{\bar{\lambda}}(x, x) \geqslant 0,
$$

where $H_{\bar{\lambda}}$ is the regular part of $G_{\bar{\lambda}}$, that is,

$$
G_{\bar{\lambda}}(a, x)=|x-a|^{-1}-H_{\bar{\lambda}}(a, x) .
$$


Now, using Lemma 4.2, we see that the assumptions of Theorem 3.1 are satisfied and therefore we can use the assertions of Theorems 3.1 and 4.1. To this aim, we introduce the following function

$$
W_{\lambda}(x)=u_{\lambda}\left(a_{\lambda, 1}\right) u_{\lambda}(x), \quad x \in \Omega .
$$

Using (4.6) and Theorem 4.1, we derive that $W_{\lambda}$ is bounded in each compact set $K$ of $\Omega \backslash\left\{\bar{a}_{1}, \bar{a}_{2}\right\}$, where $\bar{a}_{i}=$ $\lim _{\lambda \rightarrow \bar{\lambda}(\Omega)} a_{\lambda, i}$. Thus $W_{\lambda}$ converges to some function $\bar{w}$ in $C_{\mathrm{loc}}^{2}\left(\Omega \backslash\left\{\bar{a}_{1}, \bar{a}_{2}\right\}\right)$ and $\bar{w}$ satisfies

$$
\left\{\begin{array}{l}
-\Delta \bar{w}=\bar{\lambda}(\Omega) \bar{w} \quad \text { in } \Omega \backslash\left\{\bar{a}_{1}, \bar{a}_{2}\right\}, \\
\bar{w}=0 \text { on } \partial \Omega, \\
\bar{a}_{1} \text { and } \bar{a}_{2} \text { are two nonremovable singularities of } \bar{w} .
\end{array}\right.
$$

Therefore there exist $M_{1}>0$ and $M_{2}>0$ such that

$$
\bar{w}(x)=M_{1} G_{\bar{\lambda}}\left(\bar{a}_{1}, x\right)-M_{2} G_{\bar{\lambda}}\left(\bar{a}_{2}, x\right) .
$$

For $x$ close to $\bar{a}_{1}$, we have

$$
-M_{1} H_{\bar{\lambda}}\left(\bar{a}_{1}, x\right)-M_{2} G_{\bar{\lambda}}\left(\bar{a}_{2}, x\right)=-M_{1} H_{\bar{\lambda}}\left(\bar{a}_{1}, \bar{a}_{1}\right)-M_{2} G_{\bar{\lambda}}\left(\bar{a}_{2}, \bar{a}_{1}\right)+\gamma(x),
$$

where $\gamma$ is a $C^{1}$-function defined in the neighborhood of $\bar{a}_{1}$ and satisfies $\gamma\left(\bar{a}_{1}\right)=0$.

Observe that, by (4.9) and the fact that $G_{\bar{\lambda}}>0$ we have

$$
\gamma_{0}:=-M_{1} H_{\bar{\lambda}}\left(\bar{a}_{1}, \bar{a}_{1}\right)-M_{2} G_{\bar{\lambda}}\left(\bar{a}_{2}, \bar{a}_{1}\right)<0 .
$$

Applying now Pohozaev Identity, see Theorem 1.1 of [13], we have for $r$ small and $B_{r}=B\left(a_{\lambda, 1}, r\right)$

$$
\lambda \int_{B_{r}} u_{\lambda}^{2}-r \int_{\partial B_{r}}\left(\frac{u_{\lambda}^{6}}{6}+\frac{\lambda u_{\lambda}^{2}}{2}\right)=\int_{\partial B_{r}} B\left(r, x, u_{\lambda}, \nabla u_{\lambda}\right),
$$

where $B$ is the operator defined by (3.19).

Multiplying (4.13) by $u_{\lambda}^{2}\left(a_{\lambda, 1}\right)$, we obtain

$$
\lambda \int_{B_{r}} W_{\lambda}^{2}-\frac{r}{6 u_{\lambda}^{4}\left(a_{\lambda, 1}\right)} \int_{\partial B_{r}} W_{\lambda}^{6}-\frac{r \lambda}{2} \int_{\partial B_{r}} W_{\lambda}^{2}=\int_{\partial B_{r}} B\left(r, x, W_{\lambda}, \nabla W_{\lambda}\right) .
$$

Using (4.6), we derive that

$$
\begin{aligned}
& \int_{B_{r}} W_{\lambda}^{2} \leqslant \int_{B_{r}} \frac{c_{2}}{\left|y-a_{\lambda, 1}\right|^{2}} \leqslant c_{2}^{\prime} r, \\
& r \int_{\partial B_{r}} W_{\lambda}^{2} \leqslant c_{2}^{\prime} r, \\
& \frac{r}{u_{\lambda}^{4}\left(a_{\lambda, 1}\right)} \int_{\partial B_{r}} W_{\lambda}^{6} \leqslant \frac{c}{r^{3} u_{\lambda}^{4}\left(a_{\lambda, 1}\right)} \rightarrow 0 \quad \text { as } \lambda \rightarrow \bar{\lambda}(\Omega) .
\end{aligned}
$$

Thus, passing to the limit when $\lambda \rightarrow \bar{\lambda}(\Omega)$ and using (4.15)-(4.17), (4.14) becomes

$$
\int_{\partial B_{r}} B(r, x, \bar{w}, \nabla \bar{w})=\mathrm{O}(r) .
$$

We now observe that, since $\bar{w}(x)=M_{1}\left|x-\bar{a}_{1}\right|^{-1}+\gamma_{0}+\gamma(x)$, with $\gamma\left(\bar{a}_{1}\right)=0$ by direct computation we get

$$
B(r, x, \bar{w}, \nabla \bar{w})=-\frac{M_{1}^{2} \gamma_{0}}{2 r^{2}}+\mathrm{O}\left(\frac{1}{r}\right) .
$$


Thus, using (4.12), we see that

$$
\lim _{r \rightarrow 0} \int_{\partial B_{r}} B(r, x, \bar{w}, \nabla \bar{w})=-\frac{M_{1}^{2} \gamma_{0} \omega_{3}}{2}>0
$$

which contradicts (4.18). This ends the proof of Corollary 1.2 when $\bar{\lambda}(\Omega)$ is not achieved.

\section{Acknowledgements}

This work was done while the first and the second author were visiting the Mathematics Department of the University of Roma "La Sapienza". They would like to thank the Mathematics Department for its warm hospitality and in particular Professor Massimo Grossi for his constant support.

\section{Appendix A}

In this appendix, we give the definition of isolated and isolated simple blow-up points, which were first introduced by Schoen [15,16] and used extensively by $\mathrm{Li}$ [13]. We also provide the main local blow-up analysis giving first sharp pointwise estimates to a sequence $\left(w_{\lambda}\right)$, defined by (3.14), near 0 . Then we prove that 0 is in fact an isolated simple blow-up point of $\left(w_{\lambda}\right)$, ruling out the possibility of bubbles on top of bubbles. The proof follows the same scheme as in [13]. Moreover, we need some convenient additional arguments. First, we recall some notations and assumptions stated in Section 2.

We set

$$
w_{\lambda}(y)=d_{\lambda, 1}^{1 / 2} u_{\lambda}\left(a_{\lambda, 1}+d_{\lambda, 1} y\right), \quad \text { for } y \in \widetilde{\Omega}_{\lambda,+}:=d_{\lambda, 1}^{-1}\left(\Omega_{\lambda,+}-a_{\lambda, 1}\right),
$$

where $d_{\lambda, 1}=d\left(a_{\lambda, 1}, \partial \Omega_{\lambda,+}\right), \Omega_{\lambda,+}$ is defined by (3.11), and $a_{\lambda, 1}$ is defined in Theorem 3.1.

Throughout this appendix, we assume that $d_{\lambda, 1} \rightarrow 0$ as $\lambda \rightarrow \bar{\lambda}(\Omega)$ and $w_{\lambda}$ satisfies (3.15), that is

$$
\left\{\begin{array}{l}
|y|^{1 / 2} w_{\lambda}(y) \leqslant c, \quad \text { for } y \in B(0,1), \\
w_{\lambda}(0)=d_{\lambda, 1}^{1 / 2} u_{\lambda}\left(a_{\lambda, 1}\right) \rightarrow+\infty \text { as } \lambda \rightarrow \bar{\lambda}(\Omega) .
\end{array}\right.
$$

We also denote by $\Pi$ the limit domain of $\widetilde{\Omega}_{\lambda,+}$ when $\lambda \rightarrow \bar{\lambda}(\Omega)$. Recall that $w_{\lambda}$ satisfies

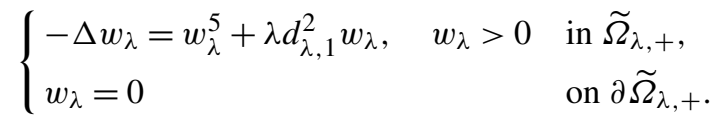

Let us now give the definition of isolated and isolated simple blow-up points.

Definition A.1. $\bar{y} \in \Pi$ is called an isolated blow-up point of $\left(w_{\lambda}\right)$ if there exist $0<\bar{r}<d(\bar{y}, \partial \Pi), C \in \mathbb{R}$ and a sequence $\left(y_{\lambda}\right)$ in $\widetilde{\Omega}_{\lambda,+}$, converging to $\bar{y}$, such that $y_{\lambda}$ is a local maximum of $w_{\lambda}, w_{\lambda}\left(y_{\lambda}\right) \rightarrow+\infty$, as $\lambda \rightarrow \bar{\lambda}(\Omega)$, and

$$
\left|y-y_{\lambda}\right|^{1 / 2} w_{\lambda}(y) \leqslant C \quad \text { for all } y \in B\left(y_{\lambda}, \bar{r}\right) .
$$

Remark A.2. (A.2) implies that 0 is an isolated blow-up point of $\left(w_{\lambda}\right)$.

To describe the behavior of blowing up solutions near an isolated blow-up point, we define spherical averages of $w_{\lambda}$ centered at $y_{\lambda}$ as follows

$$
\bar{w}_{\lambda}(r)=\frac{1}{\left|\partial B\left(y_{\lambda}, r\right)\right|} \int_{\partial B\left(y_{\lambda}, r\right)} w_{\lambda}, \quad 0<r<\bar{r} .
$$

Now we define the notion of isolated simple blow-up point.

Definition A.3. $\bar{y} \in \Pi$ is called an isolated simple blow-up point of $\left(w_{\lambda}\right)$ if $\bar{y}$ is an isolated blow-up point, such that, for some positive constant $\rho$ (independent of $\lambda$ ) the function $r^{1 / 2} \bar{w}_{\lambda}(r)$ has exactly one critical point in $(0, \rho)$ for $\lambda$ close to $\bar{\lambda}(\Omega)$. 
Now, we start giving some pointwise estimates to a sequence $\left(w_{\lambda}\right)$ near 0 . Hence forward we use $c$ to denote positive constants which may vary from formula to formula and which may depend only on $\bar{r}$.

Lemma A.4. For any $0<r<1 / 3$, we have

$$
\max _{B_{2 r} \backslash B_{r / 2}} w_{\lambda}(y) \leqslant c \min _{B_{2 r} \backslash B_{r / 2}} w_{\lambda}(y),
$$

where $c$ is some positive constant independent of $\lambda$ and $r$. Here and in the sequel, for $R>0, B_{R}$ denotes the ball $B(0, R)$.

\section{Proof. Set}

$$
\widetilde{w}_{\lambda}(y)=r^{1 / 2} w_{\lambda}(r y), \quad \text { for } y \in B_{3} .
$$

It is easy to see that $\widetilde{w}_{\lambda}$ satisfies

$$
\left\{\begin{array}{l}
-\Delta \widetilde{w}_{\lambda}=\widetilde{w}_{\lambda}^{5}+\lambda r^{2} d_{\lambda, 1}^{2} \tilde{w}_{\lambda} \quad \text { in } B_{3}, \\
0<\widetilde{w}_{\lambda}(y)<c|y|^{-1 / 2} \text { for } y \in B_{3} \backslash\{0\} .
\end{array}\right.
$$

Lemma A.4 follows immediately from Theorem 8.20 of [12].

Proposition A.5. For any $R_{\lambda} \rightarrow+\infty, \varepsilon_{\lambda}>0, \varepsilon_{\lambda} \rightarrow 0$ as $\lambda \rightarrow \bar{\lambda}(\Omega)$, we have

$$
\begin{aligned}
& \left\|w_{\lambda}^{-1}(0) w_{\lambda}\left(w_{\lambda}^{-2}(0) y\right)-\left(1+3^{-1}|y|^{2}\right)^{-1 / 2}\right\|_{C^{2}\left(B_{2 R_{\lambda}}\right)}<\varepsilon_{\lambda}, \\
& R_{\lambda} w_{\lambda}^{-2}(0) \rightarrow 0 \quad \text { as } \lambda \rightarrow \bar{\lambda}(\Omega) .
\end{aligned}
$$

Proof. It follows immediately from the fact that $w_{\lambda}^{-1}(0) w_{\lambda}\left(w_{\lambda}^{-2}(0) y\right)=v_{\lambda}$, where $v_{\lambda}$ is the function defined by (3.4).

The following proposition gives a nice property of the isolated blow-up point, which proof is contained in Proposition 2.2 of [13], up to some minor modifications.

Proposition A.6. There exists some positive constant $c$, such that,

$$
w_{\lambda}(y) \geqslant c w_{\lambda}(0)\left(1+3^{-1} w_{\lambda}^{4}(0)|y|^{2}\right)^{-1 / 2} \text { for all }|y| \leqslant 1 / 2 .
$$

Now, we state the main estimate on isolated simple blow-up points.

Proposition A.7. Assume that 0 is an isolated simple blow-up point of $\left(w_{\lambda}\right)$. Then for some positive constant $c$ independent of $\lambda$, we have

$$
w_{\lambda}(y) \leqslant c w_{\lambda}^{-1}(0)|y|^{-1} \quad \text { for all } 0<|y|<1 / 2 .
$$

Before giving the proof of Proposition A.7, we first establish the following lemma:

Lemma A.8. Assume that 0 is an isolated simple blow-up point of $\left(w_{\lambda}\right)$. Then, there exists $\alpha_{\lambda}>0, \alpha_{\lambda} \rightarrow 0, c^{\prime} R_{\lambda}^{-2} \leqslant$ $\alpha_{\lambda} \leqslant c^{\prime \prime} \log ^{-1}\left(w_{\lambda}(0)\right)$ for some positive constants $c^{\prime}$ and $c^{\prime \prime}$, such that

$$
w_{\lambda}(y) \leqslant C w_{\lambda}(0)^{-1+2 \alpha_{\lambda}}|y|^{-1+\alpha_{\lambda}} \quad \text { for all } r_{\lambda} \leqslant|y| \leqslant 1 / 2,
$$

where $r_{\lambda}=R_{\lambda} w_{\lambda}^{-2}(0)$ and $C$ is some positive constant independent of $\lambda$.

Proof of Lemma A.8. It follows from Proposition A.5 that

$$
w_{\lambda}(y) \leqslant c w_{\lambda}(0) R_{\lambda}^{-1} \text { for all }|y|=r_{\lambda} .
$$

Let $\bar{w}_{\lambda}(r)$ be defined in (A.4), it follows from the assumption of isolated simple blow-up and Proposition A.5 that there exists $\rho>0$ such that 


$$
r^{1 / 2} \bar{w}_{\lambda}(r) \text { is strictly decreasing for } r_{\lambda}<r<\rho .
$$

It follows from (A.5), (A.6) and Lemma A.4 that for $r_{\lambda}<|y|<\rho$, we have

$$
|y|^{1 / 2} w_{\lambda}(y) \leqslant c|y|^{1 / 2} \bar{w}_{\lambda}(|y|) \leqslant c r_{\lambda}^{1 / 2} \bar{w}_{\lambda}\left(r_{\lambda}\right) \leqslant c R_{\lambda}^{-1 / 2} .
$$

Therefore

$$
w_{\lambda}^{4}(y) \leqslant c R_{\lambda}^{-2}|y|^{-2} \text { for all } r_{\lambda} \leqslant|y| \leqslant \rho .
$$

Consider the following second order elliptic operator

$$
\mathcal{L}_{\lambda} \varphi:=\Delta \varphi+w_{\lambda}^{4} \varphi+\lambda d_{\lambda, 1}^{2} \varphi .
$$

Notice that $\mathcal{L}_{\lambda} w_{\lambda}=0$ and $w_{\lambda}>0$. It follows that the maximum principle holds for $\mathcal{L}_{\lambda}$.

For $0<\alpha<1$, a direct computation yields

$$
\mathcal{L}_{\lambda}\left(|y|^{-\alpha}\left(1-\lambda d_{\lambda, 1}^{2}|y|^{2}\right)\right) \leqslant-\alpha(1-\alpha)|y|^{-2-\alpha}+w_{\lambda}^{4}(y)|y|^{-\alpha} \leqslant|y|^{-2-\alpha}\left(-\alpha(1-\alpha)+c R_{\lambda}^{-2}\right),
$$

where we have used (A.7) in the last inequality.

Choosing $\alpha:=\alpha_{\lambda} \geqslant c R_{\lambda}^{-2}$, we obtain

$$
\mathcal{L}_{\lambda}\left(|y|^{-\alpha_{\lambda}}\left(1-\lambda d_{\lambda, 1}^{2}|y|^{2}\right)\right) \leqslant 0 .
$$

Another straightforward computation taking into account (A.7) shows that

$$
\mathcal{L}_{\lambda}\left(|y|^{-1+\alpha_{\lambda}}\left(1-\lambda d_{\lambda, 1}^{2}|y|^{2}\right)\right) \leqslant 0 .
$$

Set $M_{\lambda}:=\max _{\partial B_{\rho}} w_{\lambda}$ and for $r_{\lambda} \leqslant|y| \leqslant \rho$, we also set

$$
\varphi_{\lambda}(y):=2\left(M_{\lambda} \rho^{\alpha_{\lambda}}|y|^{-\alpha_{\lambda}}+A w_{\lambda}(0)^{-1+2 \alpha_{\lambda}}|y|^{-1+\alpha_{\lambda}}\right)\left(1-\lambda d_{\lambda, 1}^{2}|y|^{2}\right),
$$

where $A>1$ will be chosen later.

First, it follows from (A.8) and (A.9) that $\varphi_{\lambda}$ satisfies $\mathcal{L}_{\lambda} \varphi_{\lambda} \leqslant 0$ in the annulus $r_{\lambda} \leqslant|y| \leqslant \rho$. Secondly, by the choice of $M_{\lambda}$ and the fact that $d_{\lambda, 1} \rightarrow 0$ as $\lambda \rightarrow \bar{\lambda}(\Omega)$, we derive that

$$
\varphi_{\lambda}(y) \geqslant M_{\lambda} \geqslant w_{\lambda}(y) \text { for all }|y|=\rho .
$$

We also have

$$
\varphi_{\lambda}(y) \geqslant A w_{\lambda}(0)^{-1+2 \alpha_{\lambda}}|y|^{-1+\alpha_{\lambda}} \geqslant A w_{\lambda}(0) R_{\lambda}^{-1} \text { for all }|y|=r_{\lambda} .
$$

According to (A.5), we can choose $A$ to be sufficiently large and have

$$
\varphi_{\lambda}(y) \geqslant w_{\lambda}(y) .
$$

Applying the maximum principle in the annulus, we obtain

$$
w_{\lambda}(y) \leqslant \varphi_{\lambda}(y) \text { for all } r_{\lambda} \leqslant|y| \leqslant \rho .
$$

It follows from (A.6), (A.10) and Lemma A.4 that for any $r<\theta<\rho$, we have

$$
\rho^{1 / 2} M_{\lambda} \leqslant c \rho^{1 / 2} \bar{w}_{\lambda}(\rho) \leqslant c \theta^{1 / 2} \bar{w}_{\lambda}(\theta) \leqslant 2 c \theta^{1 / 2}\left(M_{\lambda} \rho^{\alpha_{\lambda}} \theta^{-\alpha_{\lambda}}+A w_{\lambda}(0)^{-1+2 \alpha_{\lambda}} \theta^{-1+\alpha_{\lambda}}\right) .
$$

Thus

$$
\rho^{1 / 2} M_{\lambda}\left(1-2 c \theta^{-\alpha_{\lambda}+1 / 2} \rho^{\alpha_{\lambda}-1 / 2}\right) \leqslant 2 c A \theta^{\alpha_{\lambda}-1 / 2} w_{\lambda}(0)^{-1+2 \alpha_{\lambda}} .
$$

Choose $\theta$ such that

$$
2 c(\theta / \rho)^{-\alpha_{\lambda}+1 / 2} \leqslant 1 / 2 \text { and } c^{\prime} \rho \leqslant \theta \leqslant \rho,
$$

for some positive constant $c^{\prime}$ independent of $\lambda$, we derive that

$$
M_{\lambda} \rho^{\alpha_{\lambda}}|y|^{-\alpha_{\lambda}} \leqslant C w_{\lambda}(0)^{-1+2 \alpha_{\lambda}}|y|^{-1+\alpha_{\lambda}}, \quad \text { for all } r_{\lambda} \leqslant|y| \leqslant \rho .
$$

Lemma A.8 follows from (A.10), (A.11) and Lemma A.4. 
Now, we are able to give the proof of Proposition A.7.

Proof of Proposition A.7. The inequality in Proposition A.7 for $|y|<r_{\lambda}$ follows immediately from Proposition A.5. Let $e \in \mathbb{R}^{3},|e|=1 / 2$ and set

$$
\underline{w}_{\lambda}(y)=w_{\lambda}^{-1}(e) w_{\lambda}(y) \text { for } y \in B_{2 / 3} .
$$

It is easy to see $\underline{w}_{\lambda}$ satisfies

$$
-\Delta \underline{w}_{\lambda}=w_{\lambda}^{4}(e) \underline{w}_{\lambda}^{5}+d_{\lambda, 1}^{2} \lambda \underline{w}_{\lambda} \quad \text { in } B_{2 / 3} .
$$

It follows from Lemma A.4 and standard elliptic theories that $\underline{w}_{\lambda}$ converges in $C_{\mathrm{loc}}^{2}\left(B_{2 / 3} \backslash\{0\}\right)$ to some positive function $\underline{w} \in C_{\text {loc }}^{2}\left(B_{2 / 3} \backslash\{0\}\right)$. Notice that $w_{\lambda}(e) \rightarrow 0$ due to Lemma A.8. Now, since $d_{\lambda, 1} \rightarrow 0$, we see that $\underline{w}$ satisfies

$$
-\Delta \underline{w}=0 \quad \text { in } B_{2 / 3} \backslash\{0\} .
$$

In addition, $\underline{w}$ has to have a singularity at $y=0$. Indeed Lemma A.4 and standard elliptic theories imply that, for $0<r<2 / 3$, we have

$$
\lim _{\lambda \rightarrow \bar{\lambda}(\Omega)} w_{\lambda}^{-1}(e) r^{1 / 2} \bar{w}_{\lambda}(r)=r^{1 / 2} \bar{w}_{1}(r),
$$

where $\bar{w}_{1}(r)=\left|\partial B_{r}\right|^{-1} \int_{\partial B_{r}} \underline{w}$. Therefore it follows from the definition of isolated simple blow-up that $r^{1 / 2} \bar{w}_{1}(r)$ is nonincreasing for $0<r<\rho$, which is impossible if $\underline{w}$ is regular near the origin. We derive that

$$
\underline{w}(y)=a_{1}|y|^{-1}+b_{1}(y),
$$

where $a_{1}>0$ and $b_{1}$ is some regular harmonic function in $B_{2 / 3}$. We first establish the inequality in Proposition A.7 for $|y|=1 / 2$. Namely, we prove that

$$
w_{\lambda}(e) \leqslant c w_{\lambda}^{-1}(0) \text {. }
$$

Arguing by contradiction, we suppose that (A.13) does not hold. Then, we can assume that

$$
w_{\lambda}(e) w_{\lambda}(0) \rightarrow+\infty \quad \text { as } \lambda \rightarrow \bar{\lambda}(\Omega) .
$$

Multiply (A.3) by $w_{\lambda}^{-1}(e)$ and integrate on $B_{1 / 2}$, we have

$$
-\int_{\partial B_{1 / 2}} \frac{\partial}{\partial v}\left(\underline{w}_{\lambda}\right)=-w_{\lambda}^{-1}(e) \int_{B_{1 / 2}} \Delta w_{\lambda}=w_{\lambda}^{-1}(e) \int_{B_{1 / 2}} w_{\lambda}^{5}+w_{\lambda}^{-1}(e) \int_{B_{1 / 2}} \lambda d_{\lambda, 1}^{2} w_{\lambda} .
$$

On one hand, it follows from Lemma A.4, standard elliptic theories and the harmonicity of $b_{1}$ that

$$
\lim _{\lambda \rightarrow \bar{\lambda}(\Omega)} \int_{\partial B_{1 / 2}} \frac{\partial}{\partial v}\left(\underline{w}_{\lambda}\right)=\int_{\partial B_{1 / 2}} \frac{\partial}{\partial v}\left(a_{1}|y|^{-1}+b_{1}(y)\right)<0 .
$$

On the other hand, it follows from Proposition A.5 that

$$
\int_{|y| \leqslant r_{\lambda}} w_{\lambda}^{5} \leqslant c w_{\lambda}^{-1}(0) \text { and } \int_{|y| \leqslant r_{\lambda}} w_{\lambda} \leqslant c r_{\lambda}^{2} w_{\lambda}^{-1}(0) .
$$

It follows from Lemma A.8 that

$$
\int_{r_{\lambda} \leqslant|y| \leqslant 1 / 2} w_{\lambda}^{5} \leqslant c \int_{r_{\lambda} \leqslant|y| \leqslant 1 / 2}\left(\left(w_{\lambda}(0)\right)^{-1+2 \alpha_{\lambda}}|y|^{-1+\alpha_{\lambda}}\right)^{5} \leqslant c w_{\lambda}^{-1}(0) R^{5 \alpha_{\lambda}-2}
$$

and

$$
\int_{r_{\lambda} \leqslant|y| \leqslant 1 / 2} w_{\lambda} \leqslant c\left(w_{\lambda}(0)\right)^{-1+2 \alpha_{\lambda}} \leqslant c w_{\lambda}(0)^{-1},
$$

if we choose $\alpha_{\lambda}$ such that $w_{\lambda}^{\alpha_{\lambda}}(0) \leqslant c$. 
Clearly (A.14)-(A.19) lead to a contradiction. Inequality (A.13) is thus established and therefore by Lemma A.4, the inequality in Proposition A.7 follows for $\rho \leqslant|y| \leqslant 1 / 2$. To establish the inequality in Proposition A.7 for $r_{\lambda} \leqslant$ $|y| \leqslant \rho$, we only need to scale the problem and reduce it to the case $|y|=1 / 2$. Arguing by contradiction, we suppose that there exists $\tilde{y}$ such that $r_{\lambda} \leqslant|\tilde{y}| \leqslant \rho$ and

$$
\lim _{\lambda \rightarrow \bar{\lambda}(\Omega)} w_{\lambda}(\tilde{y}) w_{\lambda}(0)|\tilde{y}|=+\infty .
$$

Set

$$
\widetilde{w}_{\lambda}(y)=(2|\tilde{y}|)^{1 / 2} w_{\lambda}(2|\tilde{y}| y) .
$$

Then $\widetilde{w}_{\lambda}$ satisfies

$$
-\Delta \widetilde{w}_{\lambda}=\widetilde{w}_{\lambda}^{5}+4 \lambda d_{\lambda, 1}^{2}|\tilde{y}|^{2} \widetilde{w}_{\lambda} \quad \text { in } B_{2 / 3}
$$

and we have that 0 is an isolated simple blow-up point of $\widetilde{w}_{\lambda}$. It follows from (A.13) that

$$
\widetilde{w}_{\lambda}(0) \widetilde{w}_{\lambda}\left((2|\tilde{y}|)^{-2} \tilde{y}\right) \leqslant c .
$$

Thus

$$
\lim _{\lambda \rightarrow \bar{\lambda}(\Omega)} w_{\lambda}(\tilde{y}) w_{\lambda}(0)|\tilde{y}|<+\infty .
$$

This is a contradiction. Hence our proposition follows.

Now, we can prove that 0 is in fact an isolated simple blow-up point.

Proposition A.9. We have that

0 is an isolated simple blow-up point of $\left(w_{\lambda}\right)$.

Proof. From Proposition A.5, it follows that $r^{1 / 2} \bar{w}_{\lambda}(r)$ has precisely one critical point in the interval $0<r<r_{\lambda}$, where $r_{\lambda}=R_{\lambda} w_{\lambda}^{-2}(0)$. Arguing by contradiction, we suppose that 0 is not an isolated simple blow-up point of $\left(w_{\lambda}\right)$ and let $l_{\lambda}$ be the second critical point of $r^{1 / 2} \bar{w}_{\lambda}(r)$ which is the nearest to the one in the interval $0<r<r_{\lambda}$. We know that

$$
l_{\lambda} \geqslant r_{\lambda}, \quad \lim _{\lambda \rightarrow \bar{\lambda}(\Omega)} l_{\lambda}=0 .
$$

Set

$$
\xi_{\lambda}(y)=l_{\lambda}^{1 / 2} w_{\lambda}\left(l_{\lambda} y\right), \quad|y|<\left(2 l_{\lambda}\right)^{-1} .
$$

It is easy to see that $\xi_{\lambda}$ satisfies

$$
\begin{cases}-\Delta \xi_{\lambda}=\xi_{\lambda}^{5}+\lambda d_{\lambda, 1}^{2} l_{\lambda}^{2} \xi_{\lambda} & \text { in } B_{1 /\left(2 l_{\lambda}\right)}, \\ |y|^{1 / 2} \xi_{\lambda}(y) \leqslant c & \text { in } B_{1 /\left(2 l_{\lambda}\right)}, \\ \lim _{\lambda \rightarrow \bar{\lambda}(\Omega)} \xi_{\lambda}(0)=+\infty . & \end{cases}
$$

Notice that $r^{1 / 2} \bar{\xi}_{\lambda}(r)$ has precisely one critical point in $0<r<1$ and

$$
\frac{\mathrm{d}}{\mathrm{d} r}\left(r^{1 / 2} \bar{\xi}_{\lambda}(r)\right)_{\mid r=1}=0, \quad \text { with } \bar{\xi}_{\lambda}(r)=\left|\partial B_{r}\right|^{-1} \int_{\partial B_{r}} \xi_{\lambda} .
$$

It follows that 0 is an isolated simple blow-up point of $\left(\xi_{\lambda}\right)$. As in the proof of (A.12), it follows that there exist some positive constant and some regular harmonic function $b \in \mathbb{R}^{3}$, such that,

$$
\xi_{\lambda}(0) \xi_{\lambda}(y) \rightarrow h(y)=a|y|^{-1}+b(y) \quad \text { in } C_{\text {loc }}^{2}\left(\mathbb{R}^{3} \backslash\{0\}\right) .
$$

Notice that $h$ is positive and we have $\underline{\lim }_{|y| \rightarrow+\infty} b(y) \geqslant 0$. It follows from the maximum principle that $b(y)$ is nonnegative and hence $b(y)=b \geqslant 0$ is a constant. 
Since

$$
\frac{\mathrm{d}}{\mathrm{d} r}\left(r^{1 / 2} \xi_{\lambda}(0) \bar{\xi}_{\lambda}(r)\right)_{\mid r=1}=\xi_{\lambda}(0) \frac{\mathrm{d}}{\mathrm{d} r}\left(r^{1 / 2} \bar{\xi}_{\lambda}(r)\right)_{\mid r=1}=0,
$$

we have, by (A.22), that

$$
\frac{\mathrm{d}}{\mathrm{d} r}\left(r^{1 / 2} h(r)\right)_{\mid r=1}=0 .
$$

It follows that

$$
b=a>0 \text {. }
$$

Applying now Pohozaev Identity, see Theorem 1.1 of [13], we have for $0<r<1 / 2$

$$
\lambda d_{\lambda, 1}^{2} l_{\lambda}^{2} \int_{B_{r}} \xi_{\lambda}^{2}-r \int_{\partial B_{r}}\left(\frac{\xi_{\lambda}^{6}}{6}+\frac{\lambda d_{\lambda, 1}^{2} l_{\lambda}^{2} \xi_{\lambda}^{2}}{2}\right)=\int_{\partial B_{r}} B\left(r, x, \xi_{\lambda}, \nabla \xi_{\lambda}\right),
$$

where $B$ is the operator defined by (3.19).

Notice that, by Proposition A.7, we have for $0<r<1 / 2$

$$
\begin{aligned}
& d_{\lambda, 1}^{2} l_{\lambda}^{2} \xi_{\lambda}^{2}(0) \int_{B_{r}} \xi_{\lambda}^{2} \leqslant c r d_{\lambda, 1}^{2} l_{\lambda}^{2} \rightarrow 0 \quad \text { as } \lambda \rightarrow \bar{\lambda}(\Omega), \\
& \xi_{\lambda}^{2}(0) r \int_{\partial B_{r}} \xi_{\lambda}^{6} \leqslant c r^{-3} \xi_{\lambda}^{-4}(0) \rightarrow 0 \quad \text { as } \lambda \rightarrow \bar{\lambda}(\Omega), \\
& d_{\lambda, 1}^{2} l_{\lambda}^{2} \xi_{\lambda}^{2}(0) \int_{\partial B_{r}} \xi_{\lambda}^{2} \leqslant c l_{\lambda}^{2} d_{\lambda, 1}^{2} \rightarrow 0 \quad \text { as } \lambda \rightarrow \bar{\lambda}(\Omega) .
\end{aligned}
$$

Multiplying (A.24) by $\xi_{\lambda}^{2}(0)$ and using (A.25)-(A.27) and the homogeneity of the operator $B$, we obtain

$$
\int_{\partial B_{r}} B(r, x, h, \nabla h)=0 .
$$

On the other hand, using (A.22), (A.23) and a direct computation, we find

$$
\lim _{r \rightarrow 0} \int_{\partial B_{r}} B(r, x, h, \nabla h)=-\frac{b^{3} \omega_{3}}{2}<0
$$

which contradicts (A.28). Proposition A.9 is thus proved.

\section{References}

[1] Adimurthi, S.L. Yadava, An elementary proof of the uniqueness of positive radial solutions of a quasilinear Dirichlet problem, Arch. Rational Mech. Anal. 127 (1994) 219-229.

[2] F.V. Atkinson, H. Brezis, L.A. Peletier, Solutions d'équations elliptiques avec exposant de Sobolev critique qui changent de signe, C. R. Acad. Sci. Paris, Sér. I 306 (1988) 711-714.

[3] F.V. Atkinson, H. Brezis, L.A. Peletier, Nodal solutions of elliptic equations with critical Sobolev exponents, J. Differential Equations 85 (1990) 151-170.

[4] A. Bahri, Critical Points at Infinity in Some Variational Problems, Pitman Res. Notes Math. Ser., vol. 182, Longman Sci. Tech., Harlow, 1989.

[5] M. Ben Ayed, K. El Mehdi, M. Hammami, A nonexistence result for Yamabe type problems on thin annuli, Ann. Inst. H. Poincaré Anal. Non Linéaire 19 (2002) 715-744.

[6] H. Brezis, Some variational problems with lack of compactness, Proc. Sympos. Pure Math. 45 (1986) 165-201.

[7] H. Brezis, T. Kato, Remarks on the Schroedinger operator with singular complex potential, J. Math. Pures Appl. 58 (1979) $137-151$.

[8] H. Brezis, L. Nirenberg, Positive solutions of nonlinear elliptic equations involving critical Sobolev exponents, Comm. Pure Appl. Math. 36 (1983) 437-477.

[9] L. Caffarelli, B. Gidas, J. Spruck, Asymptotic symmetry and local behavior of semilinear elliptic equations with critical Sobolev growth, Comm. Pure Appl. Math. 42 (1989) 271-297. 
[10] M. Clapp, T. Weth, Multiple solutions for the Brezis-Nirenberg problem, Adv. Differential Equations 10 (2005) $463-480$.

[11] O. Druet, Elliptic equations with critical Sobolev exponent in dimension 3, Ann. Inst. H. Poincaré Anal. Non Linéaire 19 (2002) 125-142.

[12] D. Gilbarg, N.S. Trudinger, Elliptic Partial Differential Equations of Second Order, Grundlehren Math. Wiss., vol. 224, Springer-Verlag, Berlin, 1977.

[13] Y.Y. Li, Prescribing scalar curvature on $S^{n}$ and related topics, Part I, J. Differential Equations 120 (1995) 319-410.

[14] O. Rey, The role of the Green's function in a nonlinear elliptic equation involving critical Sobolev exponent, J. Funct. Anal. 89 (1990) 1-52.

[15] R. Schoen, Variational theory for the total scalar curvature functional for Riemannian metrics and related topics, in: Topics in Calculus of Variations, Montecatini Terme, 1987, in: Lectures Notes in Math., vol. 1365, Springer-Verlag, Berlin, 1989, pp. 120-154.

[16] R. Schoen, On the number of solutions of constant scalar curvature in a conformal class, in: H.B. Lawson, K. Tenenblat (Eds.), Differential Geometry: A Symposium in Honor of Manfredo Do Carmo, Wiley, 1991, pp. 311-320.

[17] M. Struwe, Variational Methods: Applications to Nonlinear PDE \& Hamiltonian Systems, Springer-Verlag, Berlin, 1990. 